UNIVERSITÉ DU QUÉBEC

\author{
MÉMOIRE PRÉSENTÉ À
}

L'UNIVERSITÉ DU QUÉBEC À CHICOUTIMI

COMME EXIGENCE PARTIELLE

DE LA MAITTRISE EN RESSOURCES RENOUVELABLES

\title{
PAR
}

ISABELLE LESAGE

B. Sc. en Biologie

\section{EFFETS D'UN GRADIENT DE LUMIÈRE ET DE COMPÉTITION INTRASPÉCIFIQUE SUR LA CROISSANCE ET LA MORPHOLOGIE DE LA CIME DU SAPIN BAUMIER (ABIES BALSAMEA (L.) MILL.)}




\section{Bibliothèque}

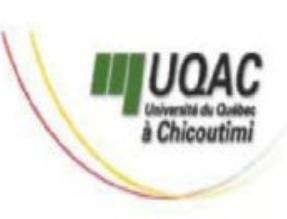

\section{Mise en garde/Advice}

Afin de rendre accessible au plus Motivated by a desire to make the grand nombre le résultat des results of its graduate students' travaux de recherche menés par ses research accessible to all, and in étudiants gradués et dans l'esprit des accordance with the rules règles qui régissent le dépôt et la governing the acceptation and diffusion des mémoires et thèses diffusion of dissertations and produits dans cette Institution, theses in this Institution, the I'Université du Québec à Université du Québec à Chicoutimi (UQAC) est fière de Chicoutimi (UQAC) is proud to rendre accessible une version make a complete version of this complète et gratuite de cette œuvre. work available at no cost to the reader.

L'auteur conserve néanmoins la The author retains ownership of the propriété du droit d'auteur qui copyright of this dissertation or protège ce mémoire ou cette thèse. thesis. Neither the dissertation or Ni le mémoire ou la thèse ni des thesis, nor substantial extracts from extraits substantiels de ceux-ci ne it, may be printed or otherwise peuvent être imprimés ou autrement reproduced without the author's reproduits sans son autorisation. permission. 


\section{RÉSUMÉ}

Cette étude caractérise la croissance et la morphologie de la cime de sapins baumiers en sous-étage en fonction de la disponibilité de la lumière en sous-bois (densité du flux photonique photosynthétisant: DFPP) et de la compétition intraspécifique. La croissance en hauteur augmente avec le pourcentage de DFPP et la hauteur $\left(R^{2}=0,633 ; p<0,001\right)$. De la même façon, la croissance radiale est corrélée avec le pourcentage de DFPP et le diamètre $\left(R^{2}=0,653 ; p<0,001\right)$. Ces deux types de croissance atteignent un maximum aux environs de $25 \%$ de DFPP. Le coefficient d'étalement, qui représente le rapport entre la flèche terminale et la longueur de la branche nodale la plus longue au premier noeud, augmente avec le pourcentage de DFPP et la hauteur de l'arbre. La compétition intraspécifique ne semble pas influencer la croissance et la morphologie de la cime des arbres recevant moins de 25\% de DFPP. On note cependant une diminution de la croissance en hauteur et et en diamètre des arbres recevant plus de $25 \%$ de DFPP lorsque la pression de compétition augmente $\left(R^{2}=0,506 ; p<0,001\right.$ et $R^{2}=0,403 ; p<0,001$ respectivement). Cette étude confirme donc l'hypothèse que le sapin baumier possède une forte plasticité morphologique. Plusieurs indicateurs de vigueur ont été développés pour déterminer directement sur le terrain si un sapin reçoit plus de $25 \%$ de DFPP, limite à laquelle l'impact de la lumière devient moins important étant donné que l'arbre ne peut utiliser une plus grande quantité de lumière. Parmi ces indicateurs, nous retrouvons, pour les arbres mesurant entre 100 et $200 \mathrm{~cm}$, un pourcentage de cime vivante supérieur à $60 \%$, un coefficient d'étalement plus grand que 1 et la présence d'au moins une branche internodale sur le dernier entre-noeud. Cependant, pour les arbres mesurant entre 50 et $100 \mathrm{~cm}$, le coefficient d'étalement est réduit à 0,75 et la présence des branches internodales est très variable. 


\section{REMERCIEMENTS}

Je désire remercier tout d'abord mon directeur de recherche, Monsieur Hubert Morin, pour l'aide qu'il a apporté à la réalisation de ce projet, mais aussi pour son ouverture à laisser place aux initiatives. J'aimerais remercier Robin Duchesneau pour son aide précieuse sur le terrain et pour son appui moral durant les expérimentations, les analyses et la rédaction de ce projet de maîtrise. Merci aussi à Germain Savard et Pierre-Yves Plourde pour l'aide technique au laboratoire. Finalement, je désire exprimer ma reconnaissance à Monsieur Christian Messier de I'UQAM pour sa participation intellectuelle tout au cours de ce projet. Ces travaux ont été rendus possible grâce à l'aide financière et support logistique dispensés par le Consortium de recherche sur la forêt boréale commerciale. 


\section{TABLE DES MATIÈRES}

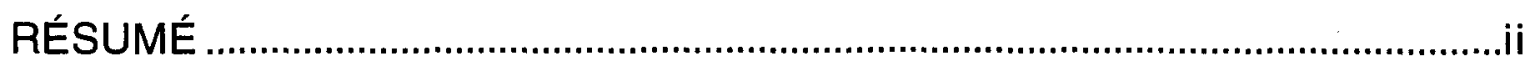

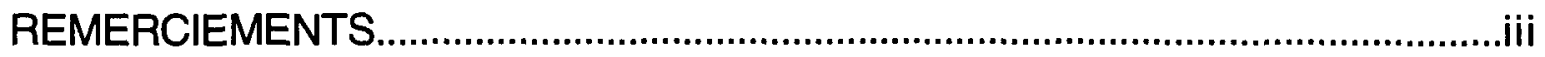

TABLE DES MATIĖRES ......................................................................................

LISTE DES TABLEAUX

LISTE DES FIGURES...................................................................................

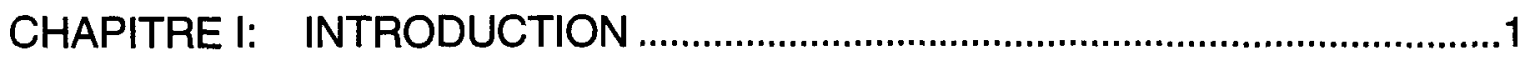

CHAPITRE II: MATÉRIEL ET MÉTHODES ....................................................5

2.1 Localisation et description de la région étudiée .................................6

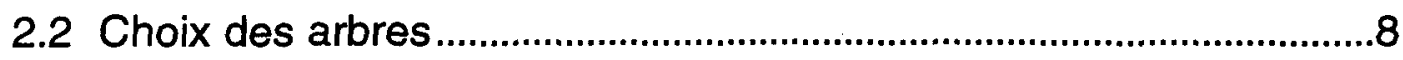

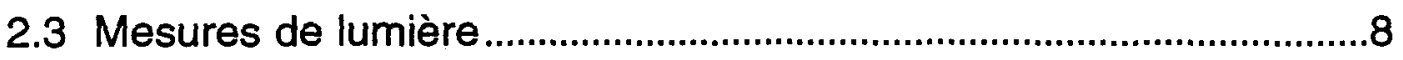

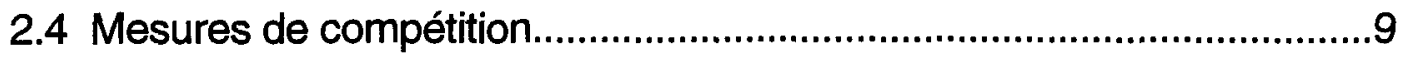

2.5 Mesures de croissance et de morphologie de la cime ......................10

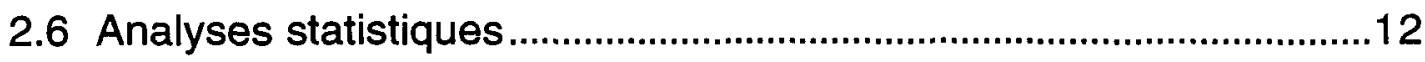

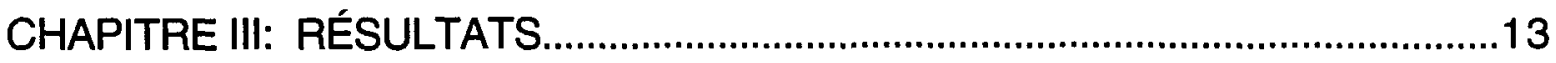

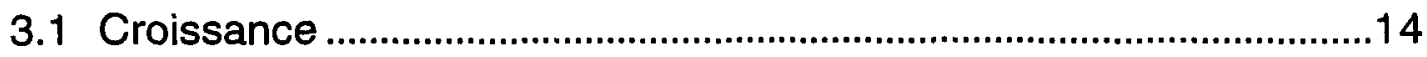

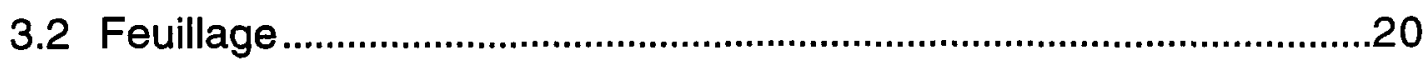

3.3 Allocation de la biomasse aérienne .................................................21

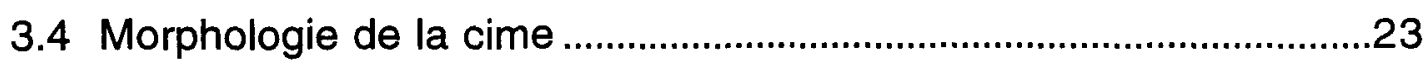

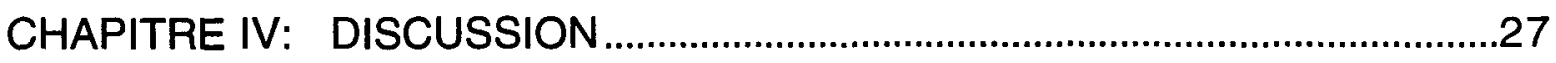

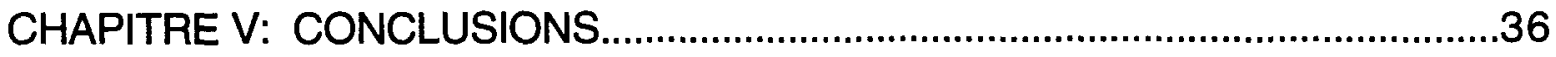

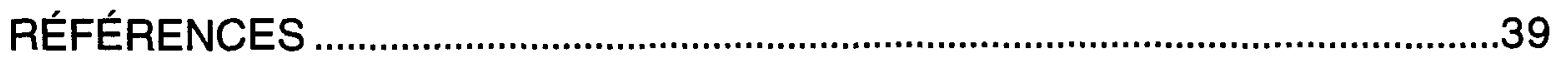




\section{LISTE DES TABLEAUX}

Tableau I: Caractéristiques générales des sites choisis...............................6

Tableau II: Indicateurs de vigueur permettant de déterminer si un sapin reçoit plus de 25\% de DFPP...............................................................35 


\section{LISTE DES FIGURES}

Figure 1: Localisation de la région étudiée ....................................................

Figure 2: Relation entre le ratio hauteur totale / diamètre à la base et le pourcentage journalier moyen de DFPP

Figure 3: Relation entre le ratio longueur des branches / poids des branches et le pourcentage journalier moyen de DFPP 16

Figure 4: Variation de (A) la croissance en hauteur relative $(\mathrm{CHR})$ et (B) la croissance radiale relative (CRR) des sapins échantillonnés en fonction du pourcentage journalier moyen de DFPP

Figure 5: Variation de (A) la croissance en hauteur relative $(\mathrm{CHR})$ et (B) la croissance radiale relative (CRR) des sapins échantillonnés recevant plus de $25 \%$ de DFPP en fonction de l'indice de compétition

Figure 6: Relation entre la masse spécifique des aiguilles et le pourcentage journalier moyen de DFPP

Figure 7: Allocation des ressources aux tissus photosynthétiques par rapport aux tissus non-photosynthétiques (en grammes) en fonction du pourcentage journalier moyen de DFPP

Figure 8: Relation entre le ratio poids des aiguilles / longueur des branches et le pourcentage journalier moyen de DFPP. .22

Figure 9: Relation entre le pourcentage de cime vivante et le pourcentage journalier moyen de DFPP 
Figure 10: Relations entre le coefficient d'étalement au noeud \#1 pour les arbres échantillonnés de 50 à $100 \mathrm{~cm}$ et de 100 à $200 \mathrm{~cm}$ et le pourcentage journalier moyen de DFPP.

Figure 11: Relation entre le coefficient d'étalement au noeud \#1 des sapins échantillonnés recevant plus de $25 \%$ de DFPP et l'indice de compétition

Figure 12: Nombre de branches internodales produites en 1996 pour les arbres échantillonnés de 50 à $100 \mathrm{~cm}$ et de 100 à $200 \mathrm{~cm}$ en fonction du pourcentage journalier moyen de DFPP 


\section{CHAPITRE I}

INTRODUCTION 
La disponibilité de la lumière affecte grandement la croissance et la morphologie de la cime de plusieurs conifères (Hellmers 1964; Logan 1969; Canham 1988; Lord et al. 1993; Klinka et al. 1992; Pacala et al. 1994; Wang et al. 1994; Chen et al. 1996). Dans le cas des conifères tolérants à l'ombre, lorsque la quantité de lumière reçue à la cime d'un arbre est faible, la croissance en hauteur s'en trouve diminuée au profit de l'expansion latérale des branches: la forme de l'arbre devient alors plus aplatie. À de fortes intensités lumineuses, c'est la croissance en longueur de la flèche terminale qui est favorisée et l'arbre prend une forme plus élancée (Kohyama 1980; Canham 1988; Parent et Messier 1995).

La compétition est un autre facteur qui peut affecter la croissance des conifères (Ruel 1992). En effet, les ressources peuvent devenir limitantes si la densité des individus dans un secteur donné devient importante (MacLean et Morgan 1983). La compétition peut donc avoir l'effet de diminuer la croissance en hauteur et en diamètre (Lavigne 1988; Frank 1990; Jobidon 1994; Takahashi 1996).

Les conifères ayant la capacité de modifier leur patron de croissance en fonction, entre autres, de la lumière reçue ont une plus grande probabilité de survie dans des milieux ombragés tel un sous-bois et, par conséquent, plus de chances de devenir des arbres dominants (O'Connell et Kelty 1994). Ces espèces sont qualifiées de tolérantes à l'ombre, c'est-à-dire qu'elles ont la capacité de croître dans des milieux restreints en lumière (Oliver et Larson 1990). Elles possèdent généralement une forte plasticité morphologique contrairement 
aux espèces dites intolérantes à l'ombre qui, elles, posséderaient plutôt une plasticité au niveau de leur physiologie (Canham 1988).

Le sapin baumier est une espèce qualifiée de très tolérante à l'ombre. II possède un bon potentiel pour s'installer et croître sous la canopée de plusieurs types de peuplements (Frank 1990). De plus, les sapins supprimés ont la capacité d'augmenter leur taux de croissance suite à une ouverture soudaine du couvert arborescent, par exemple après le passage d'une épidémie de tordeuse des bourgeons de l'épinette (Ghent 1958; Fye et Thomas 1963; Ruel et Huot 1993; Morin 1994). Leur rythme de croissance est aussi grandement affecté par la densité des arbres qui les entourent comme le démontrent plusieurs études portant sur les effets positifs d'une éclaircie (Hatcher 1964; Lavigne 1988; Pomminville 1993). Cependant, aucune étude n'a encore quantifié les effets conjoints de la lumière et de la compétition intraspécifique sur la croissance et la morphologie du sapin baumier en milieu naturel non perturbé.

Les objectifs de cette étude sont donc de quantifier la croissance et de caractériser la morphologie de la cime de sapins baumiers en sous-étage en fonction d'un gradient de lumière et de compétition intraspécifique dans une sapinière naturelle en zone boréale. De plus, cette étude a pour objectif secondaire de caractériser la plasticité morphologique du sapin baumier. Nous posons trois hypothèses de travail: 
1) L'augmentation de la lumière favorise la croissance en hauteur de la tige ainsi que la croissance radiale jusqu'à un niveau maximum.

2) La compétition intraspécifique diminue la croissance en hauteur et radiale.

3) Le sapin baumier possède une forte plasticité morphologique, c'està-dire qu'il peut modifier sa morphologie en fonction des différents facteurs environnementaux tels la lumière et la compétition. 
CHAPITRE III

MATÉRIEL ET MÉTHODES 


\subsection{Localisation et description de la région étudiée}

L'expérimentation s'est déroulée à environ $120 \mathrm{~km}$ au nord du lac SaintJean, près du lac Libéral (49049' $\mathrm{N} ; 72^{\circ} 36^{\prime}$ O) (fig.1). Le site a été choisi parce qu'il est un milieu naturel, c'est-à-dire qu'il n'a subi aucune perturbation d'origine anthropique. La température moyenne annuelle est de $-0,7^{\circ} \mathrm{C}$ (de $-19,4^{\circ} \mathrm{C}$ en janvier à $15,8^{\circ} \mathrm{C}$ en juillet), la moyenne annuelle des précipitations sous forme de pluie est de $65,1 \mathrm{~cm}$ et de $356,6 \mathrm{~cm}$ sous forme de neige (Environnement Canada 1992). Cette région se situe le domaine de dans la pessière noire à mousses de la zone boréale (Thibault 1987) et est dominée par des peuplements d'épinette noire (Picea mariana (Mill.) B.S.P.) parsemés de grands îlots de sapin baumier (Abies balsamea (L.) Mill.). Les sites étudiés étaient constitués de peuplements purs de sapin baumier sur un dépôt de till. Ils ont été choisis en fonction de l'ouverture moyenne de la canopée, de façon à avoir trois sites d'intensités lumineuses différentes. Les caractéristiques générales de ces sites sont présentées dans le tableau 1.

Tableau I: Caractéristiques générales des sites choisis (TBE: Tordeuse de bourgeons de l'épinette) (modifié de Morin et Laprise 1997)

\begin{tabular}{lccccccc}
\hline Site & $\begin{array}{c}\text { Ouverture } \\
\text { de la } \\
\text { canopée }\end{array}$ & $\begin{array}{c}\text { Année } \\
\text { moyenne } \\
\text { d'établissement } \\
\text { des arbres }\end{array}$ & $\begin{array}{c}\text { Dernière } \\
\text { épidémie } \\
\text { importante de } \\
\text { TBE ayant } \\
\text { influencé la } \\
\text { structure d'âge } \\
\text { (années) }\end{array}$ & $\begin{array}{c}\text { Structure } \\
\text { d'âge }\end{array}$ & $\begin{array}{c}\text { Densité } \\
\text { totale } \\
\text { (arbres } \geq \\
10 \mathrm{~cm})\end{array}$ & $\begin{array}{c}\text { Densité } \\
\text { de sapin } \\
\text { baumier } \\
\text { (arbres } \geq \\
10 \mathrm{~cm})\end{array}$ & $\begin{array}{c}\text { Surface } \\
\text { terrière } \\
\text { sapin } \\
\text { baumier } \\
\text { (arbres } \geq \\
10 \mathrm{~cm})\end{array}$ \\
\hline L26 & fermée & 1934 & $1944-1953$ & unimodale & 17150 & 16400 & 91 \\
L20 & moyenne & 1922 & $1944-1953$ & bimodale & 7600 & 7300 & 80 \\
L24 & ouverte & 1883 & $1909-1923$ & unimodale & 2475 & 2325 & 76 \\
\hline
\end{tabular}




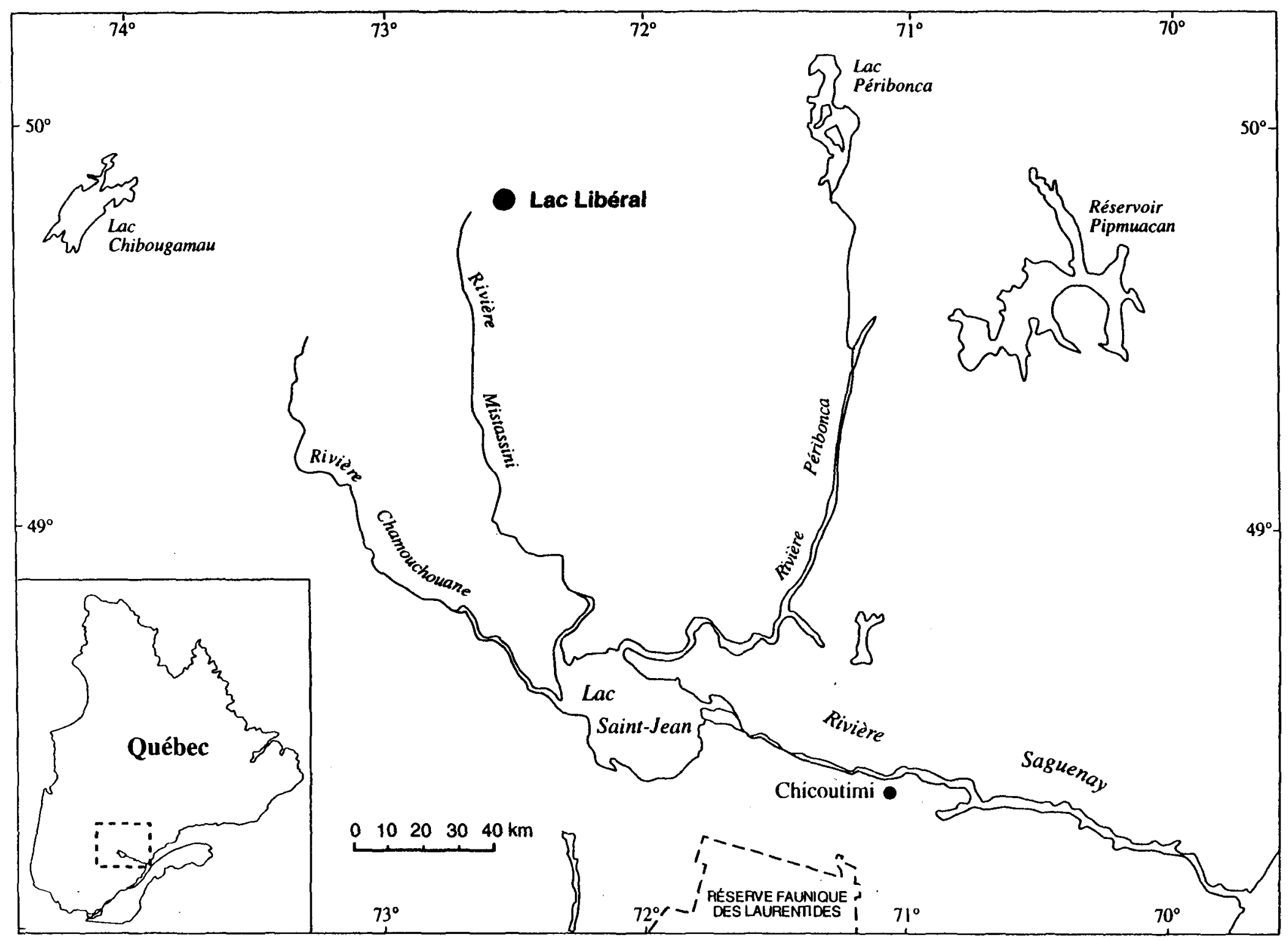

Figure 1: Localisation de la région étudiée 


\subsection{Choix des arbres}

Les sapins choisis pour l'étude ont été sélectionnés par un échantillonnage à choix raisonné (Scherrer 1984). La technique consistait à localiser à l'intérieur de chaque site trois zones d'intensités lumineuses différentes (peu, moyennement et beaucoup éclairée). À l'intérieur de chacune de ces zones, trois milieux présentant un degré de compétition intraspécifique différent (peu, moyen et beaucoup de compétition) ont été sélectionnés. Enfin, à l'intérieur de chacun de ces milieux, deux ou trois sapins en sous-étage mesurant entre 50 et $200 \mathrm{~cm}$ et ayant une flèche terminale saine ont été choisis. Trois sapins ont été choisis dans le site L24 contrairement à deux dans les sites L26 et L20 parce que l'on y retrouvait une plus grande variation de lumière et de compétition. Un total de 63 arbres a donc été récolté.

\subsection{Mesures de lumière}

La lumière reçue à la cime de chaque sapin échantillonné a été caractérisée lors de journées nuageuses selon la méthode élaborée par Messier et Puttonen (1995a) et validée par Parent et Messier (1996). Elle consiste à prendre une mesure instantanée $\left(Q_{0}\right)$ de la densité du flux photonique photosynthétisant (DFPP en $\mu \mathrm{mol}$ photons $\mathrm{m}^{-2} \mathrm{~s}^{-1}$; 400-700 $\mathrm{nm}$ ) à l'apex de la flèche terminale à l'aide d'un senseur Quantum Li-190SB (LI-COR Inc., Nebraska, États-Unis). Simultanément, la lumière incidente reçue au-dessus de la forêt $\left(Q_{i}\right)$ a été mesurée en plaçant un autre senseur Quantum Li-190SB 
couplé à un moniteur de données (Li-6200) dans un milieu découvert situé à proximité du site. Ce moniteur a été programmé pour prendre une lecture de la DFPP à toutes les cinq secondes afin de calculer une moyenne à toutes les minutes. Les valeurs absolues obtenues en milieu découvert variaient de 120 à $375 \mu \mathrm{mol}$ photons $\mathrm{m}^{-2} \mathrm{~s}^{-1}$. La DFPP mesurée à la flèche terminale a été transformée en proportion de la DFPP reçue au même moment au-dessus de la forêt, soit:

$$
\% D F P P=\left(Q_{0} / Q_{i}\right) \times 100
$$

Cette proportion représentait la moyenne journalière de lumière incidente reçue à l'apex de l'arbre étudié (Messier et Puttonen 1995a).

\subsection{Mesures de compétition}

Les mesures de la compétition intraspécifique ont été effectuées selon une méthode modifiée de Ruel (1992) élaborée pour mesurer simultanément la compétition inter- et intraspécifique. Cette méthode a été choisie parce qu'elle tenait compte des principaux paramètres concernant l'évaluation de la compétition, c'est-à-dire la hauteur de l'arbre étudié ainsi que la hauteur, la densité et la distance des arbres compétiteurs. Elle consistait premièrement à délimiter une parcelle circulaire de $1.13 \mathrm{~m}$ de rayon autour de chaque sapin échantillon et de la diviser en quatre quadrants. L'indice de compétition (I) a été calculé à l'aide de l'équation suivante:

$$
I=\frac{1}{H_{s a b}} \times \frac{1}{4} \sum_{j=1}^{4} \frac{C_{j} H_{j}}{D_{j}}
$$


où $\mathrm{H}_{\mathrm{sab}}$ est la hauteur de l'apex à la base (surface du substrat) du sapinéchantillon, $C_{j}$ est le pourcentage de recouvrement des sapins compétiteurs dans le quadrant $\mathrm{j}, \mathrm{H}_{\mathrm{j}}$ est la hauteur moyenne des sapins compétiteurs dans le quadrant $j$ et $D_{j}$ est la distance moyenne entre les sapins compétiteurs et le sapin échantillon dans le quadrant $\mathbf{j}$.

\subsection{Mesures de croissance et de morphologie de la cime}

Les sapins-échantillons ont été récoltés au mois d'octobre 1996, c'est-àdire après la fin de la saison de croissance, et rapportés au laboratoire afin de mesurer les différents paramètres de croissance suivants: la hauteur totale (de l'apex à la base du substrat), la longueur de la flèche terminale, la croissance radiale de la dernière année (épaisseur du dernier cerne à la base du substrat), le diamètre de la tige à la base et le nombre de cernes à la base. Ces données ont servi à calculer la croissance en hauteur relative (CHR) qui est la longueur de la flèche terminale divisée par la hauteur totale et la croissance radiale relative (CRR) qui est la croissance radiale de la dernière année divisée par le diamètre.

Pour chaque sapin, un échantillon de 40 aiguilles a été récolté de façon aléatoire partout sur l'arbre afin de mesurer la longueur, largeur et surface foliaire projetée à l'aide d'un analyseur d'image à balayage horizontal (MacNEEDLE, Régent Instruments, inc., Québec). Ces aiguilles ont été séchées à l'étuve et pesées afin de calculer la masse spécifique moyenne par unité de surface (mg $\mathrm{mm}^{-2}$ ). La totalité des aiguilles ainsi que les branches et le tronc pour chaque sapin ont été séchés à l'étuve puis pesés afin de calculer les ratios d'allocation 
de la biomasse suivants: la longueur des branches / poids des branches, la surface d'aiguilles par unité de branche, le ratio tissus photosynthétiques: tissus non-photosynthétiques (poids des aiguilles / poids des branches et du tronc), ainsi que le poids des aiguilles / longueur des branches et la surface d'aiguilles / longueur des branches.

La caractérisation de la morphologie de la cime s'est effectuée en calculant le pourcentage de la cime vivante (longueur de la cime vivante divisée par la hauteur totale) et en dénombrant le nombre de branches nodales par verticille et le nombre de branches internodales entre chaque verticille. De plus, le coefficient d'étalement, qui indique l'importance de la dominance apicale par rapport à la dominance latérale, a été calculé pour les huit premiers noeuds de la façon suivante:

$$
C e_{i}=\sum_{i=1}^{8} f_{i} / B L_{i}
$$

où $\mathrm{Ce}_{j}$ est le coefficient d'étalement au noeud $\mathrm{i}, \Sigma \mathrm{f}_{\mathrm{i}}$ est la somme des longueurs des entre-noeuds jusqu'au noeud $i$ et $B L_{i}$ est la longueur de la branche nodale la plus longue au noeud i. Cette équation a été modifiée de Parent et Messier (1995) qui utilisaient la moyenne des longueurs des branches nodales au noeud i plutôt que $B L_{i}$. Ce dernier paramètre semble plus pertinent au niveau biologique étant donné qu'il représente l'étalement horizontal maximal et qu'il minimisera les erreurs dues aux bris des branches (C. Messier, comm. pers.). 


\subsection{Analyses statistiques}

Des modèles de régressions linéaires et non linéaires ont été effectués afin d'expliquer les relations entre les différents paramètres étudiés et le pourcentage de DFPP, la hauteur ainsi que la compétition (Sokal et Rohlf 1981; Systat 1992). Des analyses de covariance ont été effectuées pour tester I'homogénéité des courbes des coefficients d'étalement du noeud 1 à 8 ainsi que des deux classes de hauteur (50 à $100 \mathrm{~cm}$ et 100 à $200 \mathrm{~cm}$ ) pour le coefficient d'étalement au noeud 1 (Systat 1992). 
CHAPITRE III

\section{RÉSULTATS}




\subsection{Croissance}

Les premières analyses ont démontré qu'il n'y a pas de relation entre la hauteur des sapins échantillonnés et le pourcentage de DFPP. L'âge moyen des sapins échantillonnés (par un décompte des cernes de croissance à la base) était de 22 ans $\left(S_{x}=9,6\right)$. Ceci représente l'âge minimum, car il est possible que, dans certains cas, le sapin ne produise pas de cernes à la base pendant plusieurs années (Kohyama 1980; Morin et Laprise 1997).

Aucune relation n'a pu être trouvée entre le ratio hauteur: diamètre et la DFPP (fig.2). Cependant, la grosseur ou la densité des branches semble augmenter avec la quantité de lumière disponible, car le ratio longueur des branches: poids des branches est légèrement plus faible dans les milieux les plus dégagés $\left(R^{2}=0,132 ; p=0,003 ;\right.$ fig.3).

La croissance de la flèche terminale augmente de façon significative avec la DFPP et la hauteur totale du semis selon l'équation de régression suivante: Flèche $=4,276 \ln$ DFPP $+0,072$ hauteur - 10,709 $\left(R^{2} \mathrm{aj}=0,633 ; p<0,001\right)$. De la même façon, la croissance radiale de la dernière année est influencée par la DFPP et le diamètre à la base du semis selon l'équation suivante: Crois.rad. = 0,290 In DFPP + 0,029 diamètre - 0,721 ( $\left.R^{2} a j=0,653 ; p<0,001\right)$. Afin de minimiser l'effet de la taille et du diamètre sur la croissance, il est donc nécessaire d'utiliser la croissance en hauteur relative $(\mathrm{CHR}=$ flèche $/$ hauteur) ainsi que la croissance radiale relative (CRR = crois.rad. / diamètre). La CHR et la CRR sont corrélées avec la DFPP jusqu'à saturation lumineuse, c'est-à-dire 
jusqu'à ce que l'arbre ne soit plus capable d'utiliser une plus grande quantité de lumière ( $R^{2}=0,604 ; p<0,001$ et $R^{2}=0,581 ; p<0,001$ respectivement; fig.4).

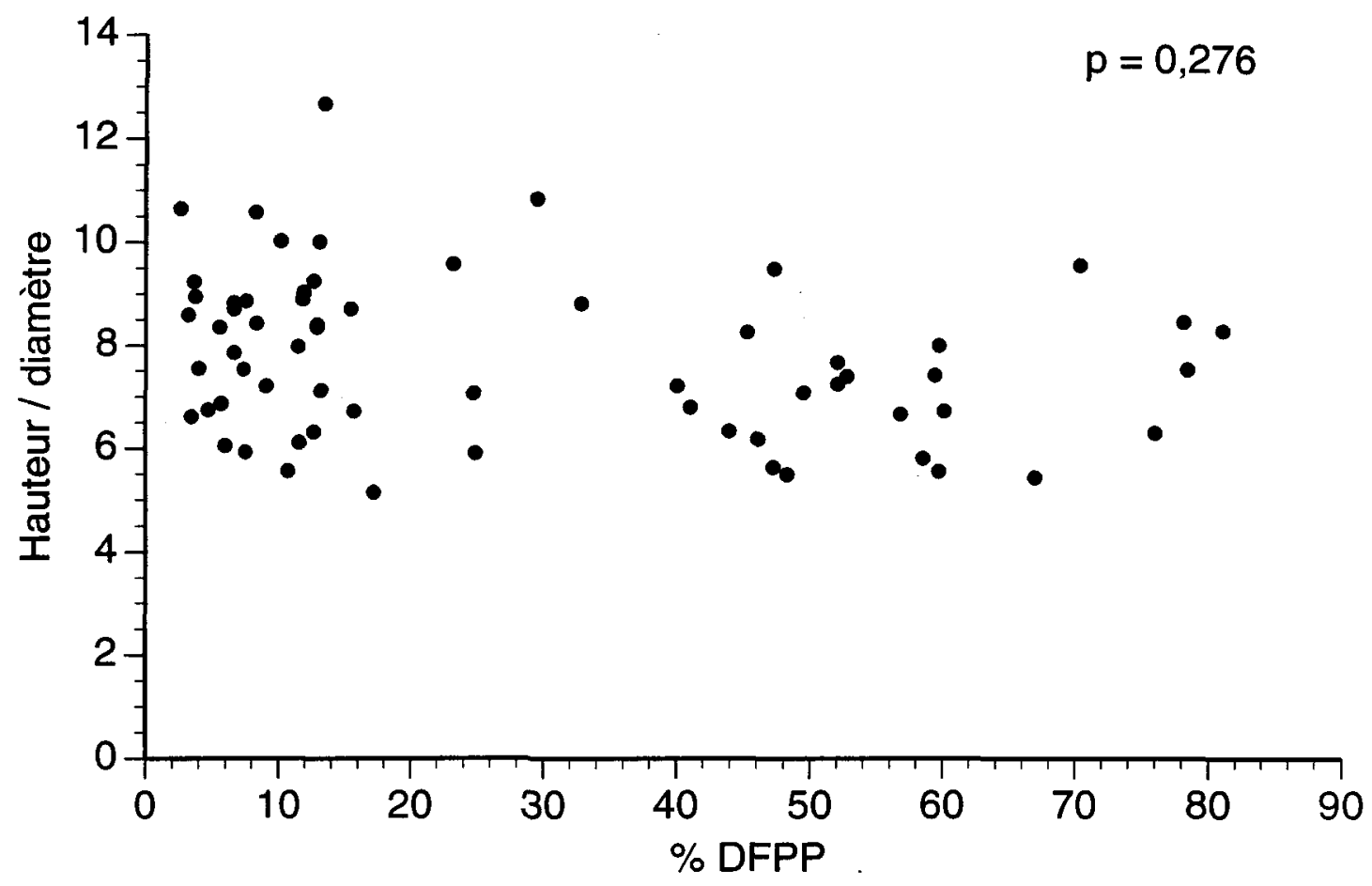

Figure 2: Relation entre le ratio hauteur totale / diamètre à la base et le pourcentage journalier moyen de DFPP 


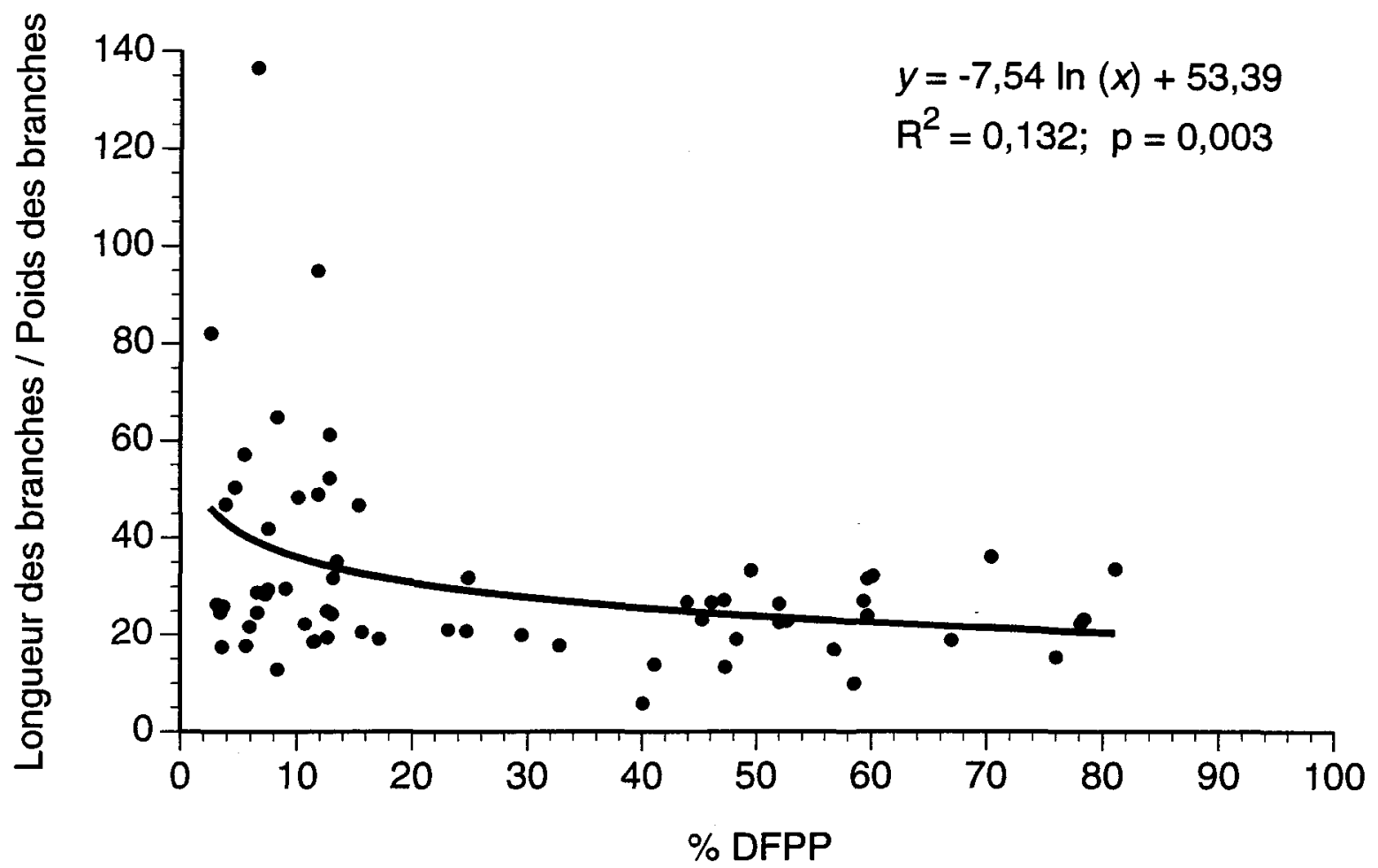

Figure 3: Relation entre le ratio longueur des branches / poids des branches $\left(\mathrm{cm} \mathrm{g}^{-1}\right)$ et le pourcentage journalier moyen de DFPP 


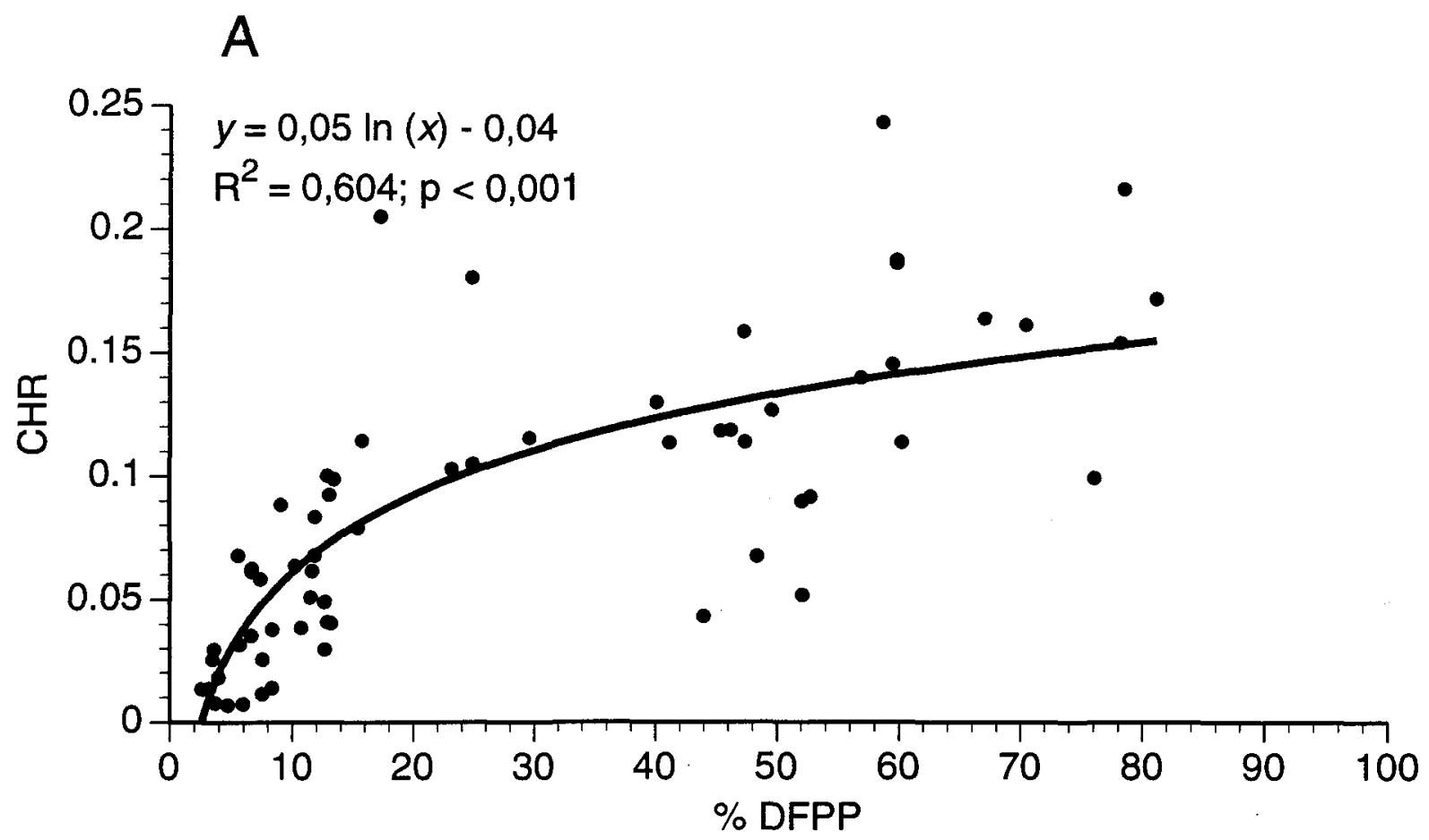

B

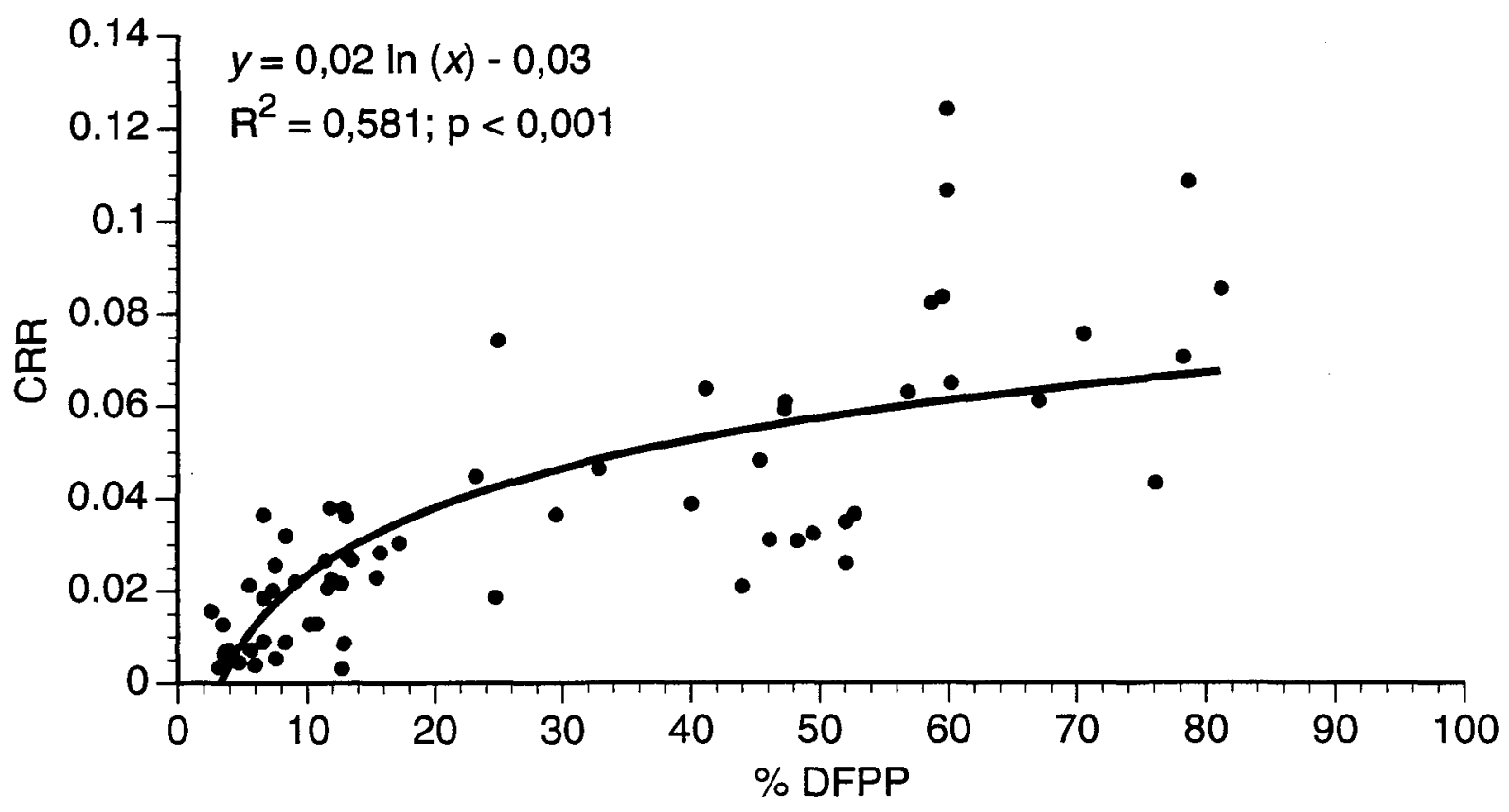

Figure 4: Variation de (A) la croissance en hauteur relative (CHR) et $(B)$ la croissance radiale relative (CRR) des sapins échantillonnés en fonction du pourcentage journalier moyen de DFPP 
En analysant l'effet de la compétition intraspécifique sur l'ensemble des arbres échantillonnés, on constate que ce paramètre n'influence pas de façon significative la $\mathrm{CHR}$ et la CRR. Afin d'obtenir la variation maximale de la croissance causée par un certain facteur, il faut que les autres facteurs environnementaux les plus influents sur la croissance soient optimaux (Hellmers 1964). Les arbres ont donc été séparés en deux classes de lumière afin de trouver une limite lumineuse à partir de laquelle il est possible de voir l'influence de la compétition sur la croissance. En effet, Chazdon (1988) a constaté qu'endessous d'une certaine limite lumineuse, les effets de la lumière sur la croissance sont trop importants pour arriver à discerner l'effet des autres facteurs biotiques et abiotiques. Dans cette étude, cette limite a été établie à $25 \%$ de DFPP, car la CHR et la CRR des arbres recevant moins de $25 \%$ de DFPP n'étaient pas influencés significativement par la compétition $(p=0,059$ et $p=0,350$ respectivement). Cependant, pour les arbres recevant plus de $25 \%$ de DFPP, la CHR et la CRR diminuaient de façon significative avec l'augmentation de la compétition $\left(R^{2}=0,506 ; p<0,001\right.$ et $R^{2}=0,403 ; p=0,002$ respectivement; fig.5). 

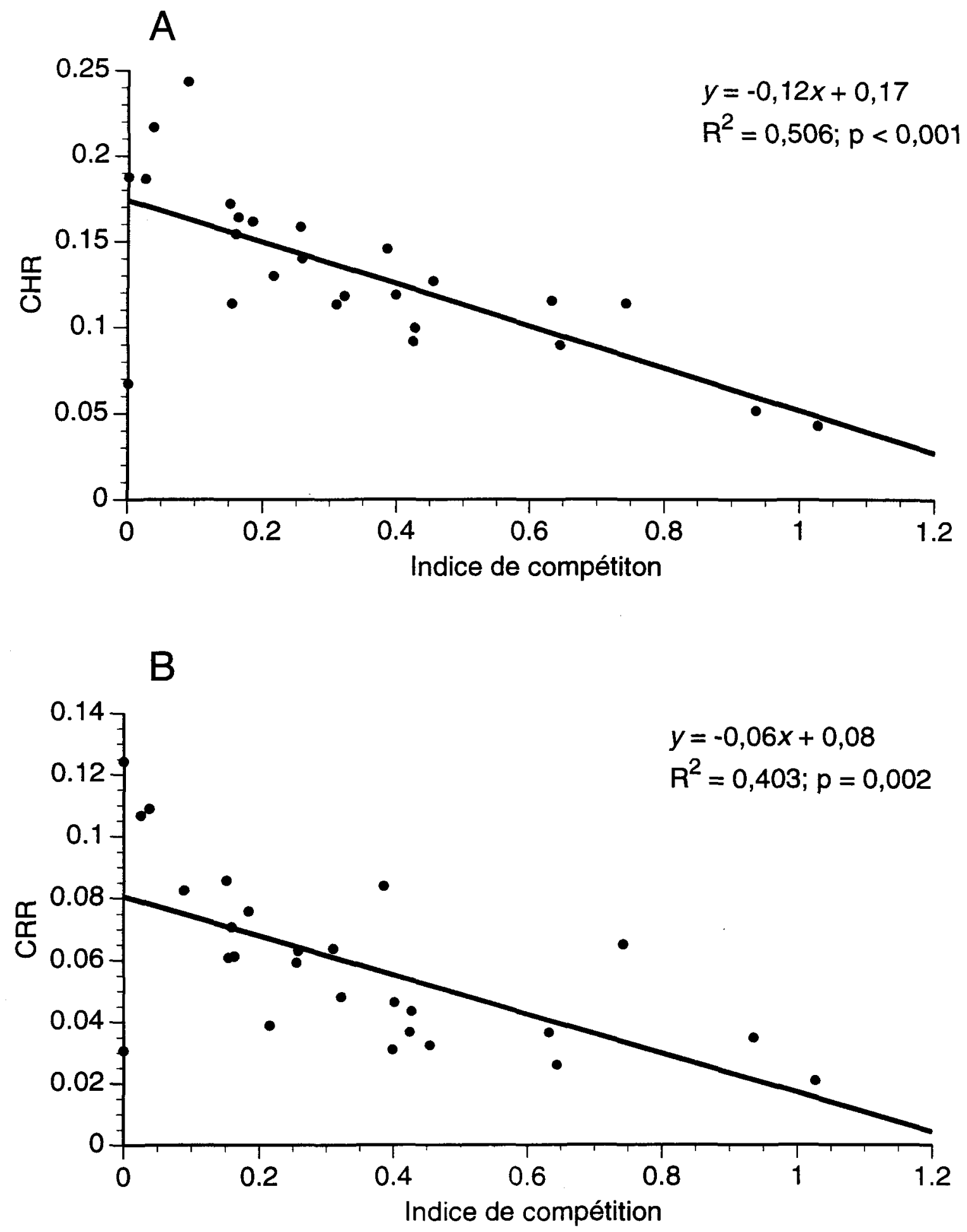

Figure 5: Variation de (A) la croissance en hauteur relative (CHR) et $(B)$ la croissance radiale relative (CRR) des sapins échantillonnés recevant plus de $25 \%$ de DFPP en fonction de l'indice de compétition 


\subsection{Feuillage}

Les analyses statistiques ont démontré qu'il n'y avait pas de relation entre la longueur, la largeur et la surface foliaire moyenne et la DFPP. De plus, ces caractéristiques des aiguilles ne variaient que très peu, ce qui suggère que ces paramètres ne sont pas plastiques. Cependant, on obtient une bonne relation allométrique entre la masse spécifique des aiguilles et la DFPP $\left(R^{2}=0,722\right.$; $p<0,001$; fig.6) et, par conséquent, c'est plutôt l'épaisseur et/ou la densité des aiguilles qui varient en fonction de la lumière.

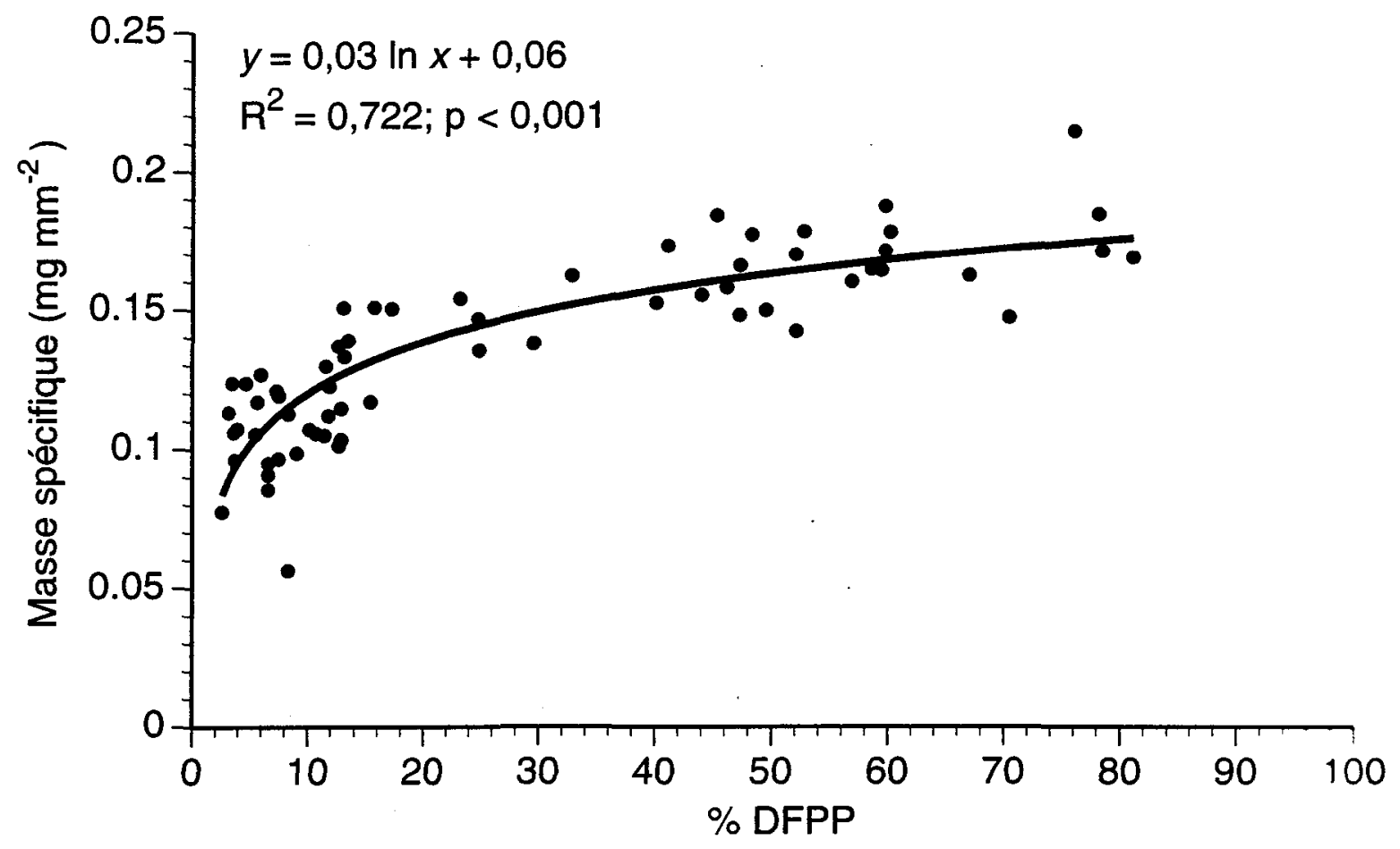

Figure 6: Relation entre la masse spécifique des aiguilles et le pourcentage journalier moyen de DFPP 


\subsection{Allocation de la biomasse aérienne}

Le meilleur ratio pour exprimer l'allocation de la biomasse aérienne chez le sapin a été le ratio tissus photosynthétiques: tissus non-photosynthétiques (poids des aiguilles / poids des branches et du tronc). Ce dernier augmente de façon logarithmique avec la lumière $\left(R^{2}=0,465 ; p<0,001 ;\right.$ fig.7). L'arbre alloue donc proportionnellement plus de ressources à ses aiguilles lorsqu'il y a plus de lumière. On retrouve aussi un plus grand poids et une plus grande surface d'aiguilles par $\mathrm{cm}$ de branche (Efficency leaf display) dans un milieu recevant plus de lumière $\left(R^{2}=0,457 ; p<0,001 ;\right.$ fig. 8); (Surface $=0,568 \ln D F P P+2,73$; $\left.R^{2}=0,132 ; p=0,003\right)$. Ces paramètres varient de la même façon que le ratio tissus photosynthétiques: tissus non-photosynthétiques, c'est-à-dire qu'ils augmentent de façon importante de 2 à $25 \%$ de DFPP, tandis qu'après $25 \%$ de DFPP, la lumière semble avoir moins d'effet. 


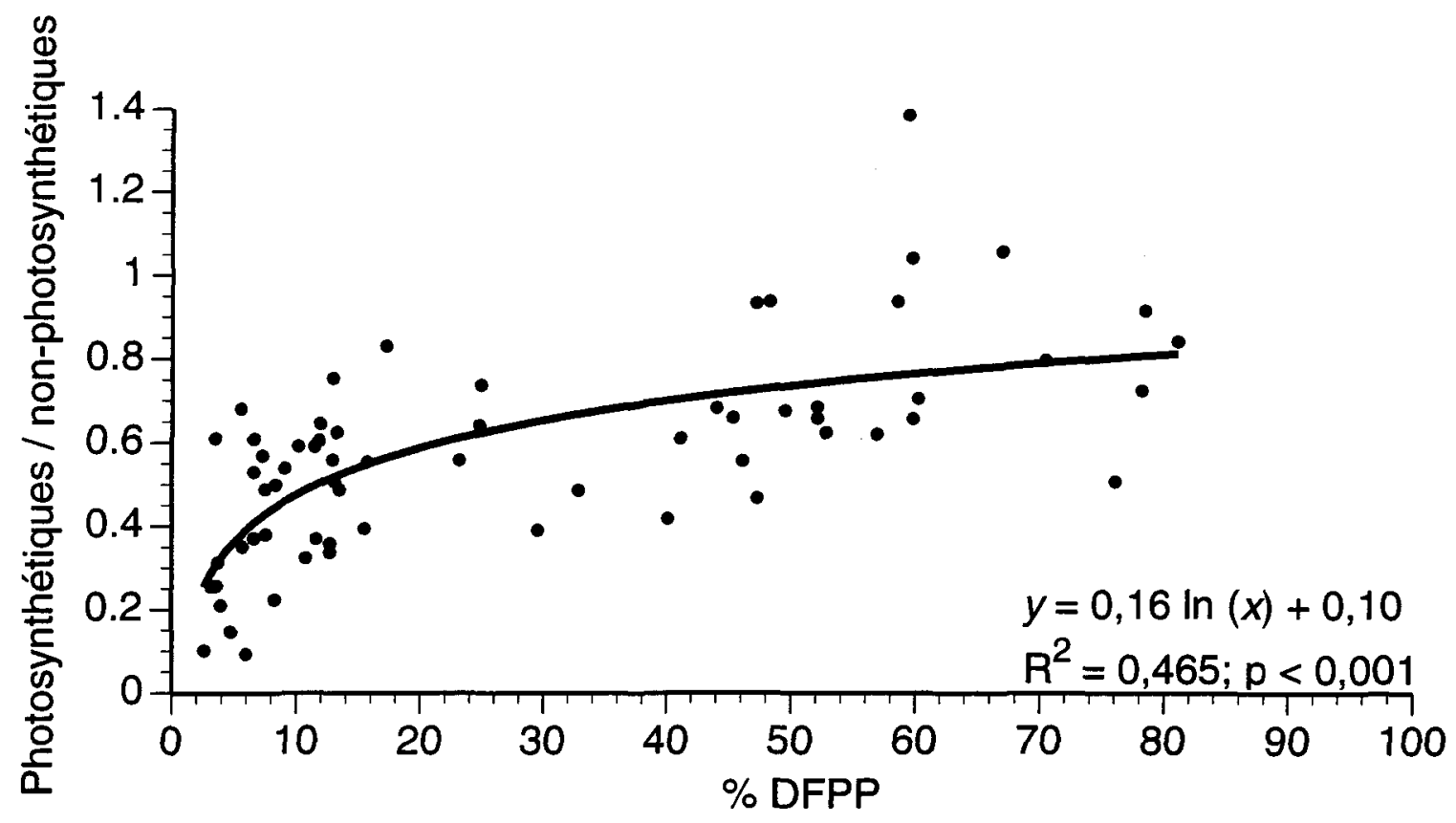

Figure 7: Allocation des ressources aux tissus photosynthétiques par rapport aux tissus non-photosynthétiques $(\mathrm{g} / \mathrm{g})$ en fonction du pourcentage journalier moyen de DFPP

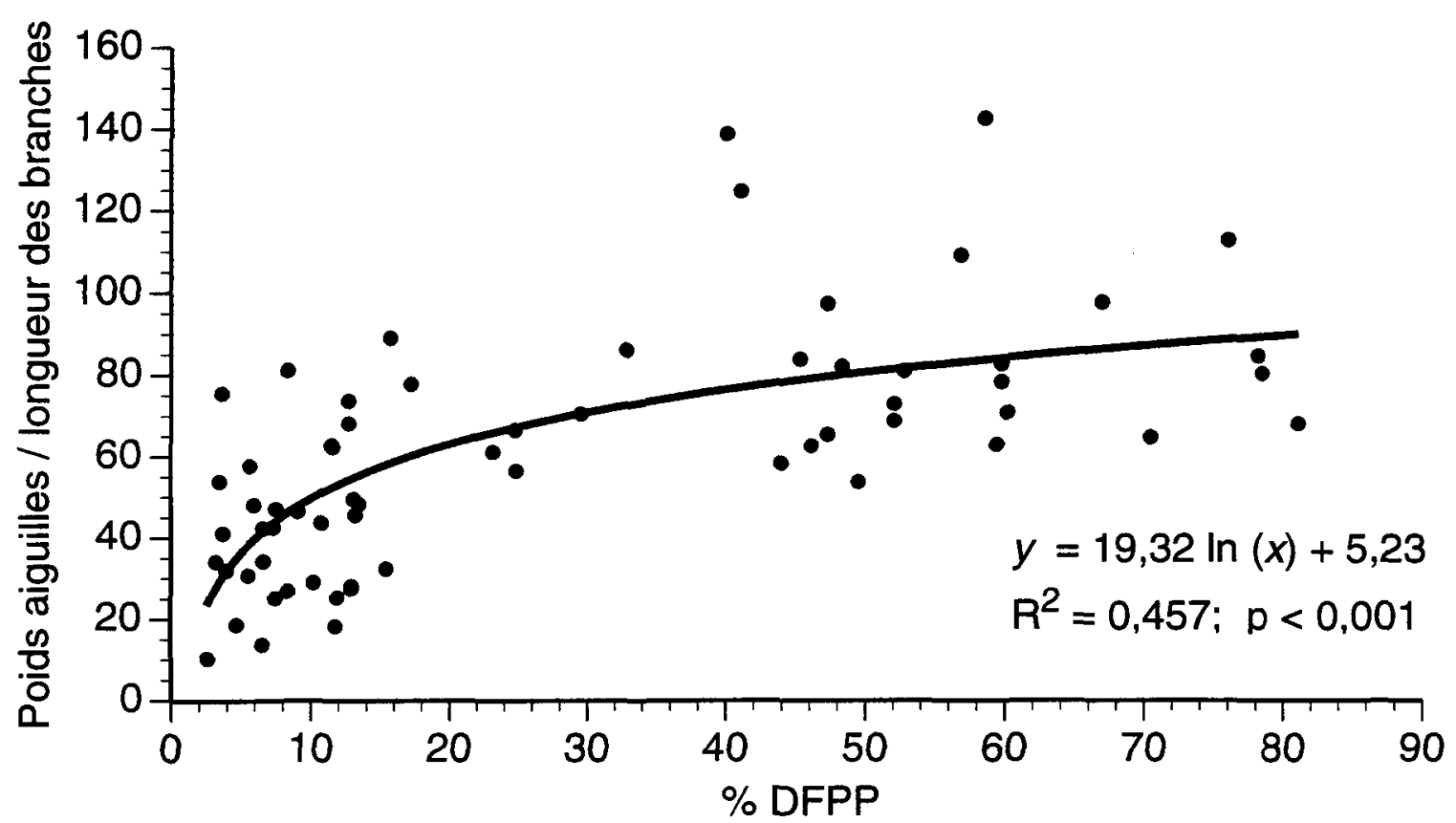

Figure 8: Relation entre le ratio du poids des aiguilles / longueur des branches et le pourentage journalier moyen de DFPP 


\subsection{Morphologie de la cime}

Le pourcentage de cime vivante augmente rapidement avec la lumière $\left(R^{2}=0,575 ; p<0,001 ;\right.$ fig. 9$)$. On remarque qu'entre 2 et $25 \%$ de DFPP, le pourcentage de cime vivante peut prendre des valeurs entre 20 et $100 \%$, alors que si la DFPP est supérieure à $25 \%$, cette valeur est d'au moins $60 \%$.

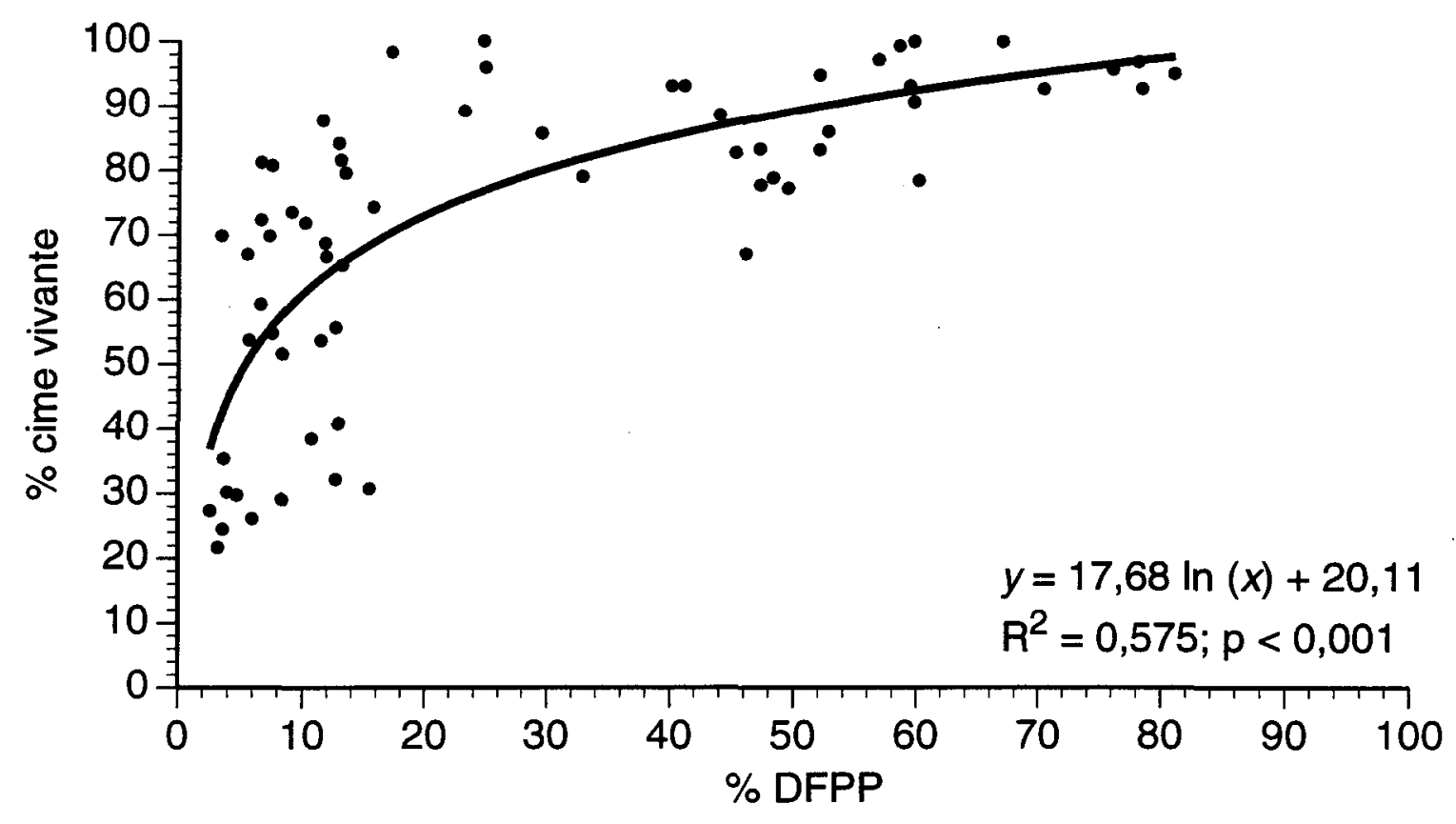

Figure 9: Relation entre le pourcentage de cime vivante et le pourcentage journalier moyen de DFPP

Le coefficient d'étalement au noeud 1 augmente avec la lumière et la hauteur (fig.10). Le transfert de dominance de la croissance des branches nodales vers une croissance à dominance apicale $(\mathrm{Ce}=1)$ est influencé par la hauteur de l'arbre. En effet, ce transfert aura lieu vers $75 \%$ de DFPP pour les 
arbres mesurant entre 50 et $100 \mathrm{~cm}$, tandis que les arbres ayant une hauteur de 100 à $200 \mathrm{~cm}$ auront une dominance apicale à partir de 25\% de DFPP. De plus, on observe que les coefficients d'étalement augmentent du noeud 1 au noeud 8 . Comme pour la croissance relative, la compétition intraspécifique diminue significativement le coefficient d'étalement au noeud 1 des arbres recevant plus de $25 \%$ de DFPP $\left(R^{2}=0,348 ; p=0,002 ;\right.$ fig. 11$)$. Les arbres recevant moins de $25 \%$ de DFPP ne semblent pas être affectés de façon significative par la compétition $(p=0,059)$.

La production de branches nodales et internodales augmente avec la lumière. Les arbres recevant moins de $5 \%$ de DFPP produisaient en moyenne une ou deux branches nodales, tandis que ceux qui recevaient plus de $25 \%$ de DFPP en produisaient de trois à cinq. Le nombre maximal de branches internodales retrouvées sur un sapin était de cinq, tandis qu'aucune branche internodale n'était produite si l'arbre recevait moins de 20\% de DFPP. Enfin, nous avons pu établir que la hauteur avait une influence sur la production de branches internodales. En effet, la majorité des sapins mesurant entre 100 et $200 \mathrm{~cm}$ produisaient au moins une branche internodale s'ils recevaient plus de $25 \%$ de DFPP, tandis que ceux mesurant entre 50 et $100 \mathrm{~cm}$ de hauteur pouvaient ne produire aucune branche internodale jusqu'au maximum de DFPP mesuré (fig.12). Contrairement à la croissance en hauteur et radiale, la compétition interspécifique ne semblait pas avoir d'effet sur la production de branches internodales, et ce, même pour les arbres recevant plus de $25 \%$ de DFPP. 


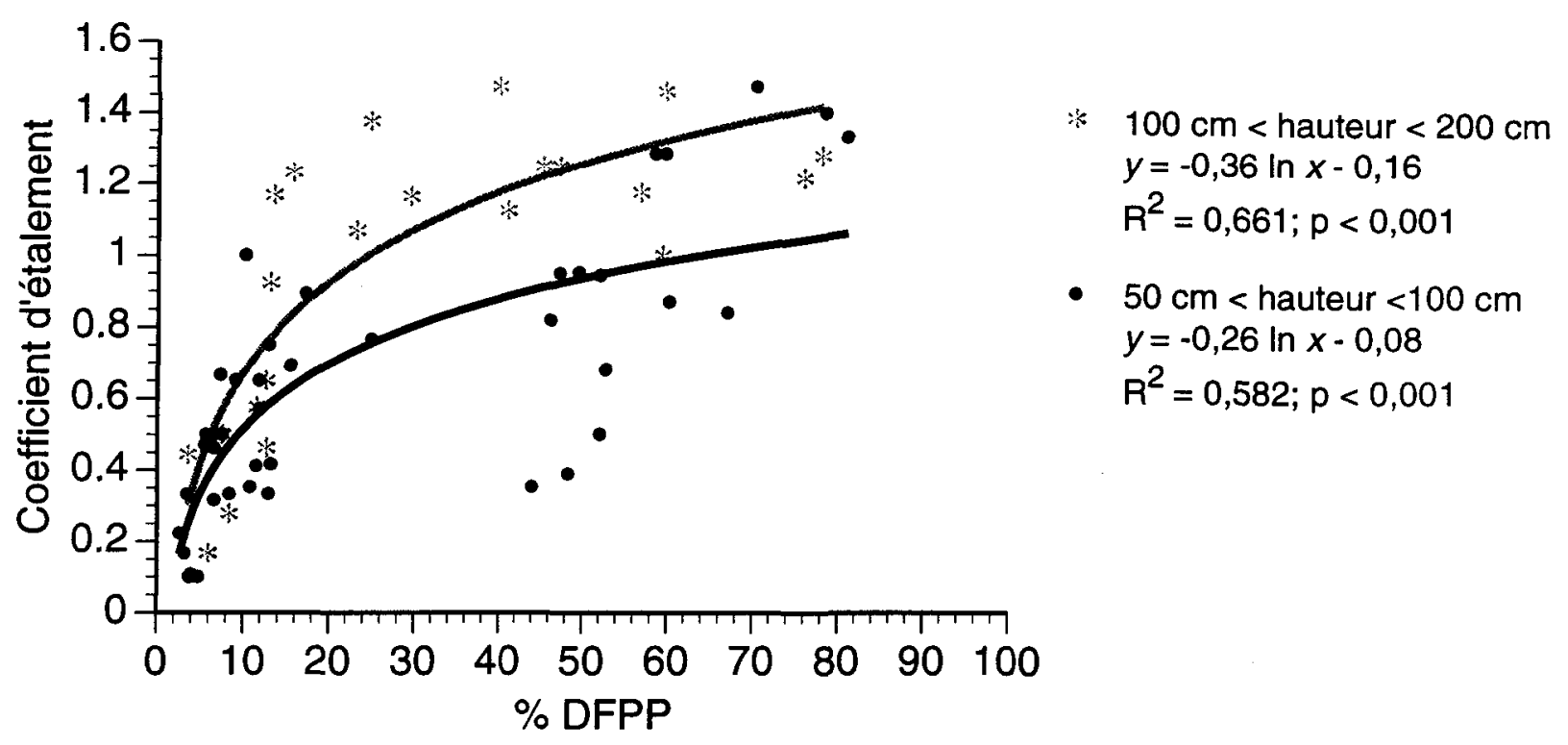

Figure 10: Relations entre le coefficient d'étalement au noeud \#1 pour les arbres échantillonnés de 50 à $100 \mathrm{~cm}$ et de 100 à $200 \mathrm{~cm}$ et le pourcentage journalier moven de DFPP (courbes différentes à $p<0,001$ )

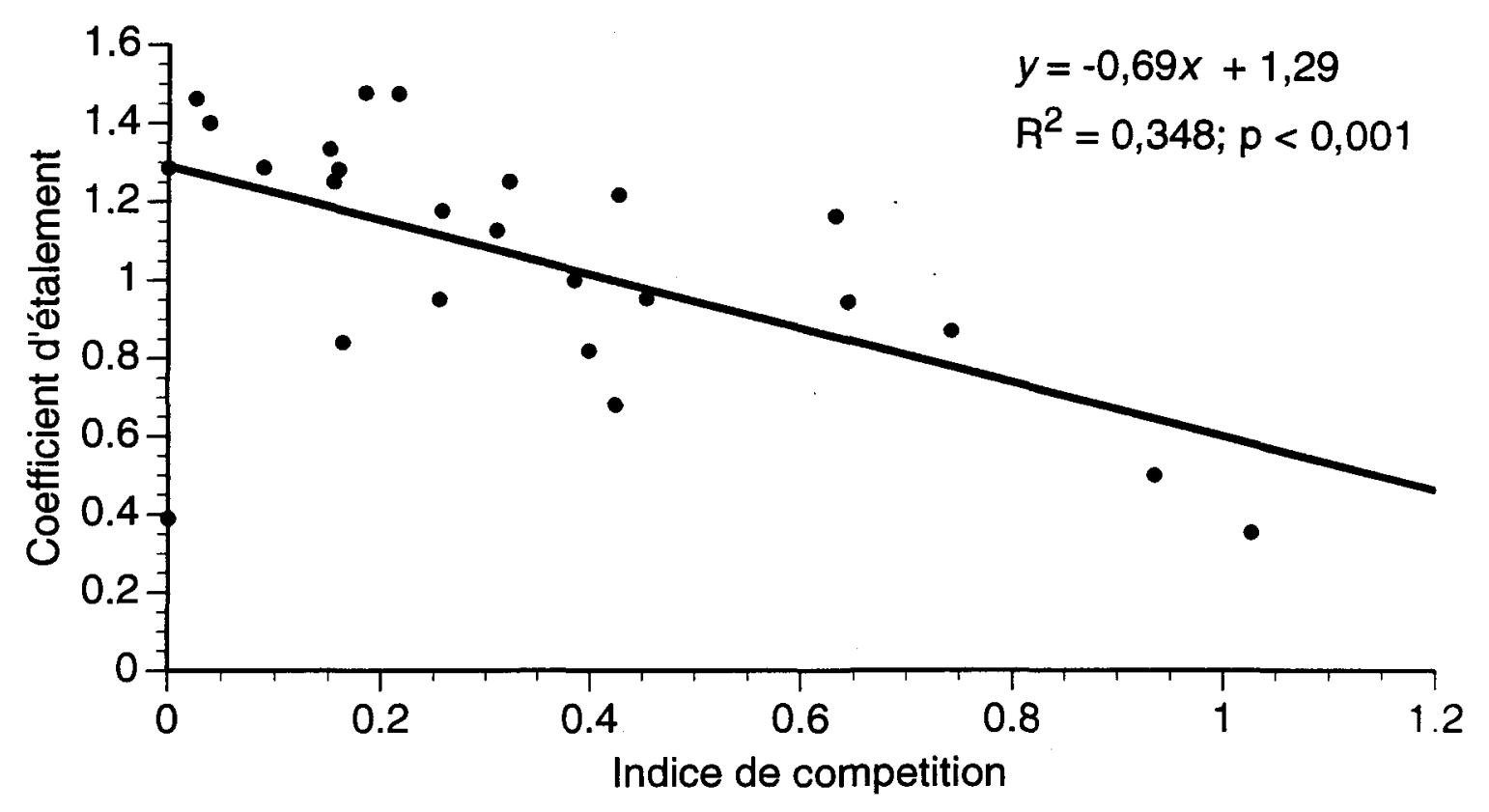

Figure 11: Relation entre le coefficient d'étalement au noeud \#1 des sapins échantillonnés recevant plus de $25 \%$ de DFPP et l'indice de compétition 


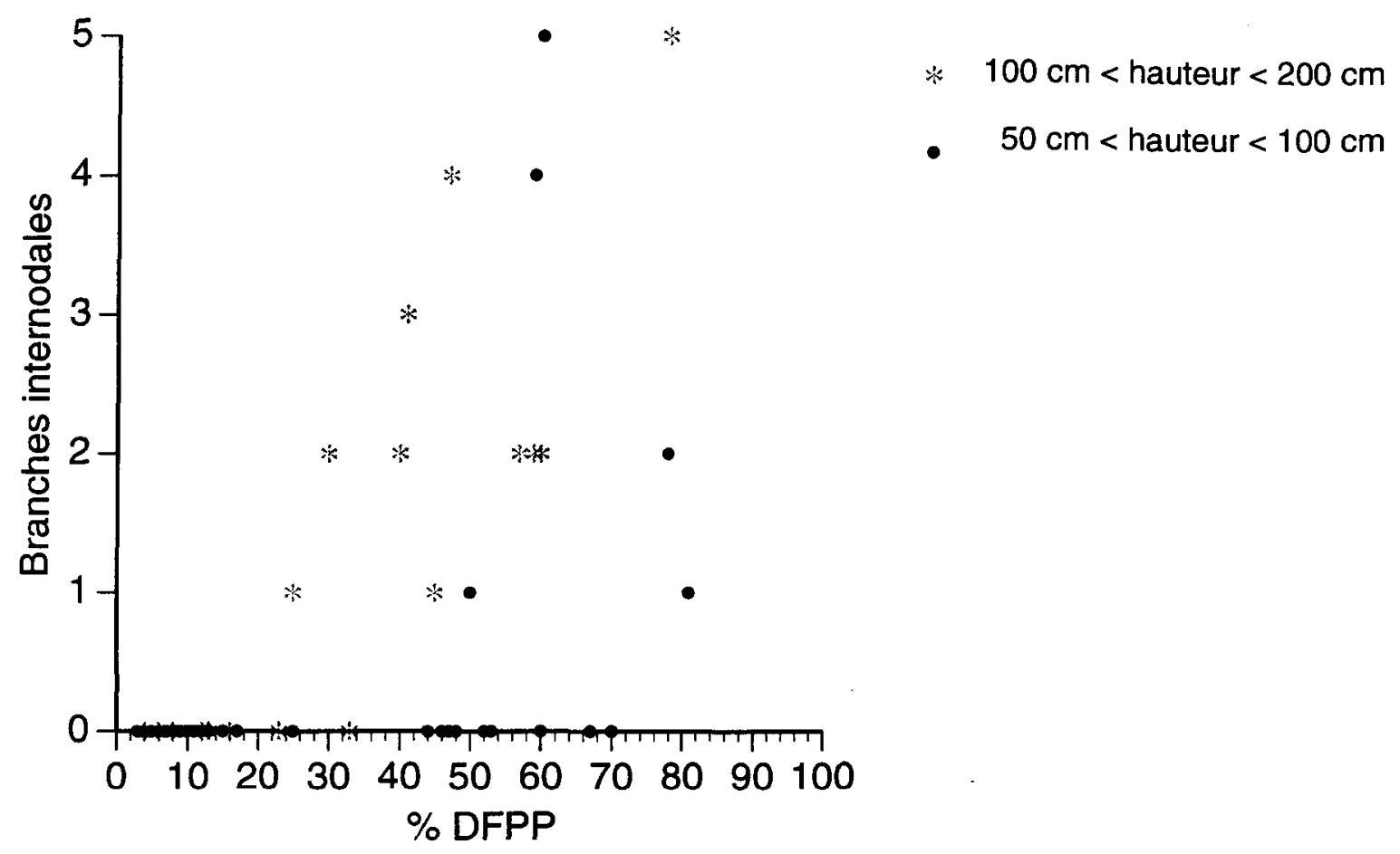

Figure 12: Nombre de branches internodales produites en 1996 pour les arbres échantillonnés de 50 à $100 \mathrm{~cm}$ et de 100 à $200 \mathrm{~cm}$ en fonction du pourcentage journalier moyen de DFPP 


\section{CHAPITRE IV}

\section{DISCUSSION}


Les résultats de cette étude confirment donc l'importance de la lumière et de la compétition sur la croissance et la morphologie de sapins baumiers en sous-étage. L'absence de relation entre la hauteur de l'arbre et la lumière peut être expliquée par le fait que la méthode d'échantillonnage consistait à prendre, dans chaque environnement lumineux, des arbres de différentes tailles afin que ce paramètre ne soit pas lié à la quantité de lumière disponible. II fut donc impossible d'isoler l'effet de l'âge sur les différents paramètres de croissance, car, pour une même taille, un sapin croissant dans un milieu ombragé était nécessairement plus âgé qu'un sapin croissant dans un milieu dégagé. On note cependant qu'une augmentation de la lumière favorise la croissance en hauteur et en diamètre jusqu'à un maximum atteint dans les environs de $25 \%$ de DFPP (Sprugel 1989). Une augmentation de la lumière entraîne une augmentation de la variabilité au niveau des paramètres de croissance, car d'autres facteurs biotiques et abiotiques commencent à influencer plus fortement la croissance (Klinka et al. 1992; Wang et al. 1994). Avant 25\% de DFPP, les effets de la lumière sont trop importants et ils masquent l'effet des autres facteurs (Chazdon 1988), ce qui explique que l'effet de la compétition n'est observable que sur les sapins recevant plus de lumière.

Wang et al. (1994) affirment que le ratio hauteur: diamètre des espèces tolérantes à l'ombre diminue avec un accroissement de la disponibilité en lumière. Dans la présente étude, il ne semble pas y avoir de plasticité au niveau de ce ratio, ces résultats corroborant ceux de Kohyama (1980) pour Abies mariesii. Cependant, on observe une certaine plasticité au niveau de la grosseur ou de la densité relative des branches, ce qui peut suggérer que les branches 
sont plus minces dans les endroits plus ombragés. Cette caractéristique permet au sapin supprimé ayant des branches relativement plus longues d'augmenter sa projection horizontale et ainsi d'avoir plus de chances d'intercepter les taches de lumière (Oliver et Larson 1990; Klinka et al. 1992; Chen et al. 1996).

Une augmentation de la lumière n'entraîne pas nécessairement une augmentation de la surface moyenne pour une aiguille comme plusieurs études l'ont démontré pour différentes espèces (McClendon et McMillen 1982; Ducrey 1992; Messier et Puttonen 1995b). Cependant, étant donné que la masse spécifique des aiguilles est moins élevée en milieu ombragé, on peut supposer que c'est au niveau de l'épaisseur et/ou de la densité des aiguilles que l'adaptation à un milieu plus restreint en lumière s'est effectuée. Selon Abrams et Kubiske (1990) et Klinka et al. (1992), la variation de la masse spécifique en fonction de la lumière serait due à une augmentation de l'épaisseur plutôt qu'à une augmentation de la densité. Dans un milieu ombragé, la moyenne d'âge des aiguilles est plus élevée parce qu'elles sont moins actives photosynthétiquement (Kohyama 1980) et elles sont plus denses étant donné que la densité des aiguilles augmente avec l'âge (Beets et Lane 1987). Par conséquent, on peut supposer que la diminution de la masse spécifique des aiguilles en milieu ombragé n'est pas due à une diminution de la densité (celle-ci semble plutôt augmenter), mais bien à une plus faible épaisseur. Des aiguilles plus minces en milieu ombragé permettent d'avoir une plus grande surface pour la même quantité de ressources investie (Fitter et Hay 1989) et elles sont plus efficaces à capter la lumière lorsqu'elle devient une ressource limitante (Jordan et Smith 1993; Chen et al. 1996). Cependant, on retrouve un plus grand nombre 
d'aiguilles par centimètre de branche dans les endroits les plus ouverts, ce qui permet de maximiser l'interception lumineuse sans augmenter la quantité de bois nécessaire au soutien (Carter et Smith 1985; Canham 1988; Leverenz et Hinckley 1990).

Le ratio tissus photosynthétiques: tissus non photosynthétiques est le meilleur ratio pour exprimer l'allocation des ressources en fonction de la lumière. Un arbre se situant dans un milieu ouvert alloue donc plus de ressources à ses aiguilles. Ces résultats sont en contradiction avec ceux de Kohyama (1980) qui a établi que le ratio feuillage: bois des gaulis de Abies mariesii est plus faible dans les milieux les plus éclairés. Cependant, l'âge de l'individu peut affecter grandement ce ratio. En effet, pour une même taille, la proportion de matériel photosynthétique sera beaucoup plus faible pour un arbre plus âgé étant donné que ce dernier perd régulièrement ses aiguilles et que ses structures de soutien augmentent avec les années.

Le pourcentage de cime vivante est aussi affecté par la quantité de lumière disponible. Dans les milieux relativement pauvres en lumière (DFPP $<25 \%$ ), la cime vivante peut prendre des valeurs entre 20 et $100 \%$. La quantité de lumière reçue à la cime du sapin ne peut donc pas prédire la profondeur de la cime qui dépend probablement plus de la quantité de lumière qui arrive à pénétrer jusqu'au bas de l'arbre. Une mesure de l'atténuation lumineuse du haut en bas de l'arbre serait donc plus efficace pour prédire le pourcentage de cime vivante. Cependant, dans les milieux où la lumière disponible est plus abondante (DFPP $>25 \%$ ), la cime vivante est au moins de 60\%. Malgré l'atténuation verticale 
de la lumière à travers l'arbre, les branches du bas parviennent à recevoir suffisamment de lumière et l'élagage naturel est moins important. Ces résultats sont similaires à ceux rapportés par O'Connell et Kelty (1994) qui ont démontré que le pourcentage de cime vivante du pin blanc est très élevé dans les milieux ouverts.

Le coefficient d'étalement au noeud 1 est influencé d'une part par la lumière et d'autre part par la hauteur du sapin (Emmingham et Waring 1973). Un arbre recevant plus de lumière sera plus élancé, mais il faudra aussi tenir compte de la hauteur, car un petit arbre peut avoir une forme aplatie même s'il reçoit une bonne quantité de lumière. Une moins grande quantité de feuillage pour les petits arbres pourrait expliquer pourquoi leur croissance en hauteur est moins importante. Cependant, il semble que la croissance des branches nodales soit moins affectée par la taille de l'arbre que la croissance de la flèche terminale. En effet, si la croissance des branches nodales était autant affectée par la taille, le coefficient d'étalement n'augmenterait pas avec la hauteur du sapin. Donc, l'augmentation du coefficient d'étalement en fonction de la hauteur de l'arbre est surtout due à une augmentation de la croissance de la flèche terminale.

Le coefficient d'étalement dans les milieux les plus éclairés est plus élevé que dans les milieux ombragés, ce qui veut dire que la croissance de la flèche terminale dans ces derniers endroits est moins importante que la croissance des branches nodales (Oliver et Larson 1990; Klinka et al. 1992; Parent et Messier 1995). En effet, un arbre supprimé ne tire que peu d'avantages à croître verticalement (sauf si la compétition est très élevée), alors que son expansion 
horizontale lui permet d'avoir une plus grande surface projetée pour intercepter la lumière (Klinka et al. 1992). En effet, le gain en carbone journalier semble être plus dépendant des taches de lumière que de la lumière diffuse reçue tout au long de la journée. De là l'importance pour les arbres supprimés de favoriser une expansion latérale au détriment de la croissance en hauteur (Emmingham et Waring 1973; Chazdon et Pearcy 1991). Enfin, de la même façon que Parent et Messier (1995) l'ont démontré pour les noeuds 1 à 4 , on remarque une augmentation du coefficient d'étalement du noeud 1 à 8 . Si l'environnement lumineux des sapins n'a pas changé depuis les huit dernières années, cette augmentation pourrait être expliquée par l'élagage partiel ainsi que par la réduction de la croissance des branches inférieures (Kohyama 1980; O'Connell et Kelty 1994). Ce phénomène peut être la conséquence de la diminution de la lumière du haut en bas de l'arbre causée par l'ombrage des branches supérieures sur les branches inférieures ou par un contrôle hormonal de l'arbre.

Le nombre de branches internodales produites en 1996 est aussi fonction de la lumière disponible et de la hauteur de l'arbre. Tous les arbres échantillonnés recevant moins de $20 \%$ de DFPP n'ont produit aucune branche nodale en 1996, toute l'énergie solaire étant utilisée pour la croissance des structures principales. Après $20 \%$ de DFPP, nous avons pu établir que la hauteur de l'arbre devient un critère important pour déterminer le débourrement des branches secondaires, ce qui n'avait pas pu être établi par Parent et Messier (1995) qui avaient les paramètres hauteur et lumière liés (petits arbres dans les milieux ombragés et grands arbres dans les milieux éclairés). En effet, les arbres de 100 à $200 \mathrm{~cm}$ de hauteur produisaient de 1 à 5 branches internodales, tandis

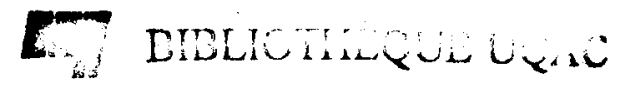


qu'il était peu fréquent que les arbres mesurant entre 50 et $100 \mathrm{~cm}$ produisent des branches internodales et ce, peu importe la quantité de lumière qu'ils recevaient. Ce phénomène peut être expliqué par le fait que les arbres de petite taille n'avaient pas une assez grande surface photosynthétique pour produire des branches supplémentaires au cours de la saison de croissance. Le nombre de branches internodales retrouvé sur les sapins échantillonnés dans cette étude est relativement faible comparé à celle effectuée par Parent et Messier (1995), ces auteurs retrouvant jusqu'à 23 branches internodales produites durant la dernière saison de croissance. De plus, dans notre étude, on n'observe aucune production de branches internodales avant $20 \%$ de DFPP, tandis que ces derniers auteurs en retrouvaient à partir d'environ $9 \%$ de DFPP. Le type de sol (dépôt argileux vs till) ainsi que la composition de la strate arborescente (mixte vs conifères) pourraient expliquer en partie ces différences. Une forêt contenant une plus grande proportion de feuillus aura aussi plus de variations annuelles au niveau de la pénétration de la lumière dans le sous-bois. Au printemps, les conifères dans le sous-bois peuvent commencer à faire de la photosynthèse bien avant que les feuillus dominants aient déployé leur feuillage et ainsi prendre une avance dans leur croissance. Ils peuvent aussi profiter d'une ouverture de la canopée à l'automne lorsque les feuilles tombent des arbres. Dans un peuplement composé essentiellement d'espèces sempervirentes, les sapins du sous-bois ne profitent pas de ces avantages et demeurent dans un milieu restreint en lumière pendant toute l'année. Par conséquent, ils ne peuvent pas toujours synthétiser suffisamment de ressources pour pouvoir les investir dans la production de branches secondaires. 
Il a été démontré que la compétition intraspécifique diminuait la croissance de la flèche terminale et le coefficient d'étalement des arbres recevant plus de $25 \%$ de DFPP. La diminution du coefficient d'étalement au noeud 1 serait attribuable en grande partie à la diminution de la croissance de la flèche terminale. II semble que la croissance de branches nodales ne soit que faiblement ou pas du tout affectée par la compétition, car le coefficient d'étalement au noeud 1 ne reste pas stable tout au long du gradient de compétition. En effet, un coefficient stable démontrerait que, pour chaque perte de croissance de la flèche terminale, il y aurait une perte similaire au niveau de la croissance des branches nodales. On peut donc affirmer que la perte de croissance due à la compétition intraspécifique au niveau de la flèche terminale est plus importante que celle au niveau des branches nodales (Takahashi 1996).

L'impact de la compétition est très difficile à quantifier si l'on emploie des indices de compétition (méthode non destructrice) par rapport à l'éclaircie des arbres compétiteurs (méthode destructrice). En effet, si la densité des arbres dans un secteur donné est faible (compétition faible), c'est possiblement parce que le site n'est pas très propice à l'installation des semis et à la croissance des arbres. De la même façon, si la régénération est très dense (forte compétition), c'est probablement parce que la qualité du site est supérieure à cet endroit et une bonne croissance des semis s'ensuit (Berkowitz et al. 1995). Dans plusieurs études, comme celle dont il est présentement question, l'objectif est de quantifier la croissance d'un semis en fonction de l'intensité de la compétition qui l'entoure. Cependant, l'effet du "bon site" contrebalance en partie l'effet de la compétition qui se traduit par une réduction de la croissance (Berkowitz et al. 1995). Par 
conséquent, il est pratiquement impossible de séparer la densité des arbres de la qualité du site. L'impact de la compétition s'en trouve souvent sous-estimé.

Même si la prise de données pour quantifier la DFPP reçue à la cime de l'arbre est relativement simple et efficace, il n'est pas toujours possible de se procurer les instruments de mesure nécessaires et on ne peut pas toujours attendre une journée complètement nuageuse. II devient donc intéressant de développer des indicateurs de vigueur afin de déterminer si un sapin donné reçoit suffisamment de lumière pour bien croître. Les indicateurs qui sont présentés dans le tableau II peuvent donc servir à déterminer dans la majorité des cas si un sapin donné reçoit plus de $25 \%$ de DFPP, limite au-delà de laquelle l'impact de la lumière devient moins importante.

Tableau II: Indicateurs de vigueur permettant de déterminer si un sapin reçoit plus de $25 \%$ de DFPP

\begin{tabular}{ccc}
\hline Indicateurs de vigueur & $\begin{array}{c}\text { Sapins mesurant entre } \\
100 \text { et } 200 \mathrm{~cm}\end{array}$ & $\begin{array}{c}\text { Sapins mesurant entre } \\
50 \text { et } 100 \mathrm{~cm}\end{array}$ \\
\hline $\begin{array}{c}\text { Proportion de la flèche } \\
\text { terminale (Flèche/hauteur) }\end{array}$ & $\geq 10 \%$ & $\geq 10 \%$ \\
$\begin{array}{c}\text { Proportion de la cime vivante } \\
\text { (cime/hauteur totale) }\end{array}$ & $\geq 60 \%$ & $\geq 60 \%$ \\
Coefficient d'étalement & $\geq 1,00$ & $\geq 0,75$ \\
$\begin{array}{c}\text { Branches internodales } \\
\text { (sur le dernier entre-noeud) }\end{array}$ & Au moins une & - \\
\hline
\end{tabular}




\section{CHAPITRE V}

\section{CONCLUSIONS}


Les résultats de cette étude démontrent que la croissance en hauteur des sapins baumiers en sous-étage augmente avec la hauteur et la lumière jusqu'à un niveau maximum. De la même façon, la croissance radiale augmente avec le diamètre et la lumière. De plus, le coefficient d'étalement augmente avec la quantité de lumière disponible, c'est-à-dire que la proportion de la flèche terminale par rapport à la longueur de la branche nodale la plus longue au premier noeud augmente de façon logarithmique avec la DFPP. Cependant, ce même coefficient est aussi influencé par la hauteur de l'arbre, ce qui fait qu'un petit sapin aura une forme plus aplatie qu'un sapin plus grand s'ils reçoivent la même quantité de lumière.

D'un autre côté, la compétition intraspécifique ne semble pas influencer la croissance et la morphologie de la cime des arbres recevant moins de $25 \%$ de DFPP où la lumière est le facteur le plus important. On note cependant une diminution de la croissance (en hauteur et radiale) des arbres recevant plus de $25 \%$ de DFPP lorsque la pression de compétition augmente. De plus, la compétition semble affecter plus la croissance de la flèche terminale que la croissance des branches nodales au noeud 1 étant donné que le coefficient d'étalement augmente de la même façon que la croissance de la flèche terminale.

À la lumière de ces conclusions, on peut affirmer que la sapin possède un patron de croissance différent selon la lumière disponible, sa hauteur et la compétition intraspécifique l'entourant. II est donc juste d'affirmer que le sapin baumier possède une forte plasticité au niveau de la morphologie de sa cime. 
Enfin, des études traitant de l'atténuation de la lumière du haut en bas de l'arbre sont nécessaires pour mieux comprendre l'effet de la lumière et de la compétition sur la plasticité au niveau de la morphologie de la cime (Jobidon 1994). II serait aussi intéressant de traiter les mêmes paramètres de croissance sur des sapins installés sous un couvert de feuillus afin de mieux comprendre l'impact des variations lumineuses au cours d'une saison de croissance. 


\section{RÉFÉRENCES}

Abrams, M.D. et Kubiske, M.E. 1990. Leaf structural characteristics of 31 hardwood and conifer tree species in central Wisconsin: influence of light regime and shade-tolerance rank. For. Ecol. Manage., 31: 245-253.

Beets, P.N. et Lane, P.M. 1987. Specific leaf area of Pinus radiata as influenced by stand age, leaf age and thinning. N. Z. J. For. Sci. 17: 283-291.

Berkowitz, A.R., Canham, C.D. et Kelly, V.R. 1995. Competition vs. facilitation of tree seedling growth and survival in early successional communities. Ecology 76: 1156-1168.

Canham, C.D. 1988. Growth and canopy architecture of shade-tolerant trees: response to canopy gaps. Ecology 69: 786-795.

Carter, G.A. et Smith, W.K. 1985. Influence of shoot structure on light interception and photosynthesis in conifers. Plant physiol., 79: 1038-1043.

Chazdon, R.L. 1988. Sunflecks and their importance to forest understory plants. Adv. Ecol. Res. 18: 1-63.

Chazdon, R.L. et Pearcy, R.W. 1991. The importance of sunflecks for forest understory plants. BioScience 41: 760-766.

Chen, H.Y.H., Klinka, K. et Kayahara, G.J. 1996. Effects of light on growth, crown architecture, and specific leaf area for naturally established Pinus contorta var. latifolia and Pseudotsuga menziesii var. glauca saplings. Can. J. For. Res., 26: 1149-1157.

Ducrey, M. 1992. Variation in leaf morphology and branching pattern of some tropical rain forest species from Guadeloupe (French West Indies) under semi-controlled light conditions. Ann. Sci. For. 49: 553-570.

Emmingham, W.H. et Waring, R.H. 1973. Conifer growth under different light environments in the Siskiyou mountains of southweatern Oregon. Northwest Science 47: 88-99.

Environnement Canada 1992. Sommaire météorologique mensuel, Chibougamau-Chapais, Serv. Envir. Atmos., Gouv. du Can., janvierdécembre 1992. 
Fitter, A.H. et Hay, R.K.M. 1989. Environmental physiology of plants. Academic Press. London.

Frank, R.M. 1990. Abies balsamea (L.) Mill. - Balsam fir. Dans Sylvics of north America. Vol.1 Conifers. Éditeurs: R.M. Burns et B.H. Honkala. U.S. Dep. Agric. Agric. Handb. no654.

Fye, R.E. et Thomas, J.B. 1963. Regeneration of balsam fir and spruce about fifteen years following release by spruce budworm attack. For. Chron. 39: 385-397.

Ghent, A.W. 1958. Studies of regeneration of forest stands devastated by the budworm. II. Age, height growth and related studies of balsam fir seedlings. For. Sci. 3: 184-208.

Hatcher, R.J. 1964. Balsam fir advance growht after cutting in Québec. For. Chron. 40: 86-92.

Hellmers, H. 1964. Distribution of growth in tree seedlings stems as affected by temperature and light. Dans The formation of wood in forest trees. M.M. Zimmerman éd. Academic Press, New York. pp. 533-547.

Jobidon, R. 1994. Light threshold for optimal black spruce (Picea mariana) seedling growth and development under brush competition. Can. J. For. Res. 24: 1629-1635.

Jordan, D.N. et Smith, W.K. 1993. Simulated influence of leaf geometry on sunlight interception and photosynthesis in conifer needles. Tree Physiol. 13: $29-39$.

Klinka, K., Wang, Q., Kayahara, G.J., Carter, R.E. et Blackwell, B.A. 1992. Lightgrowth response relationships in Pacific silver fir (Abies amabilis) and subalpine fir (Abies lasiocarpa). Can. J. Bot. 70: 1919-1930.

Kohyama, T. 1980. Growth pattern of Abies mariesii saplings under conditions of open-growth and suppression. Bot. Mag. Tokyo 93: 13-24.

Lavigne, M.B. 1988. Growth and net assimilation rates in thinned and unthinned stand of balsam fir. Can. J. For. Res. 18: 1205-1210.

Leverenz, J.W. et Hinckley, T.M. 1990. Shoot structure, leaf area index and productivity of evergreen conifer stand. Tree physiol., 6: 135-149.

Logan, K.T. 1969. Growth of tree seedlings as affected by light intensity. IV. Black spruce, white spruce, balsam fir, and eastern white cedar. Can. For. Serv. Dep. Fish. For. Publ. n01256. 
Lord, D., Morissette, S. et Allaire, J. 1993. Influence de l'intensité lumineuse, de la température nocturne de l'air et de la concentration en $\mathrm{CO}_{2}$ sur la croissance de semis d'épinette noire (Picea mariana) produits en récipients en serres. Can. J. For. Res. 23: 101-110.

MacLean, D.A. et Morgan, M.G. 1983. Long-term growth and yield response of young fir to manual and chemical release from shrub competition. For. Chron. 59:177-183.

McClendon, J.H. et McMillen, G.G. 1982. The control of leaf morphology and the tolerance of shade by woody plants. Bot. Gaz. 143: 79-83.

Messier, C. et Puttonen, P. 1995a. Spatial and temporal variation in the light environment of developing Scots pine stands: the basis for a quick and efficient method of caracterizing light. Can. J. For. Res., 25: 343-354.

Messier, C. et Puttonen, P. 1995b. Growth, allocation, and morphological responses of Betula pubescens and Betula pendula to shade in developing Scots pine stands. Can. J. For. Res., 25: 629-637.

Morin, H. 1994. Dynamics of balsam fir forests in relation to spruce budworm outbreaks in the Boreal Zone of Quebec. Can. J. For. Res. 24: 730-741.

Morin, H. et Laprise, D. 1997 . Seedling bank dynamics in boreal balsam fir forests. Can. J. For. Res. Sous presse.

O'Connell, B.M. et Kelty, M.J. 1994. Crown architecture of understory and opengrown white pine (Pinus strobus L.) saplings. Tree Physiol. 14: 89-102.

Oliver, W.W. et Larson, B.C. 1990. Forest stand dynamics. McGraw-Hill, Inc. New York.

Pacala, S.W., Canham, C.D., Silander, J.A., Jr. et Kobe, R.K. 1994. Sapling growth as a function of ressources in a north temperate forest. Can. J. For. Res. 24: 2172-2183.

Parent, S. et Messier, C. 1995. Effets d'un gradient de lumière sur la croissace en hauteur et la morphologie de la cime du sapin baumier régénéré naturellement. Can. J. For. Res., 25: 878-885.

Parent, S. et Messier, C. 1996. A simple and efficient method to estimate microsite light availability under a forest canopy. Can. J. For. Res., 26: 151-154. 
Pomminville, P. 1993. Évolution quinquennale de la régénération protégée lors de récoltes par abattage manuel dans les sapinières. For. Chron. 69: 569578.

Ruel, J.-C. 1992. Impact de la compétition exercée par le framboisier (Rubus idaeus L.) et les feuillus de lumière sur la croissance du sapin (Abies balsamea (L.) Mill.) en régénération. Can. J. For. Res., 22: 1408-1416.

Ruel, J.-C. et Huot, M. 1993. Impact de la tordeuse des bourgeons de l'épinette (Choristoneura fumiferana (Clem.)) sur la régénération des sapinières après la coupe à blanc. For. Chron. 69: 163-172.

Scherrer, B. 1984. Biostatistique. Gaëtan Morin éditeur, Boucherville.

Sokal, R.R., et Rohlf, F.J. 1981. Biometry, 2e éd. W.H. Freeman and Co., New York.

Sprugel, D.G. 1989. The relationship of evergreenness, crown architecture, and leaf size. Am. Nat. 133: 465-479.

Systat. 1992. Statistics. 5e éd. Systat Inc., Evanston.

Takahashi, K. 1996. Plastic response of crown architecture to crowding in undrestorey trees of two co-dominating conifers. Annals of botany 77: 159164.

Thibault, M. 1987. Les régions écologiques au Québec méridional. Deuxième approximation. Carte. Service de la recherche. Min. de l'énergie et des ressources, Québec.

Wang, G.G., Qian, H. et Klinka, K. 1994. Growth of Thuja plicata seedling along a light gradient. Can. J. Bot. 72: 1749-1757. 